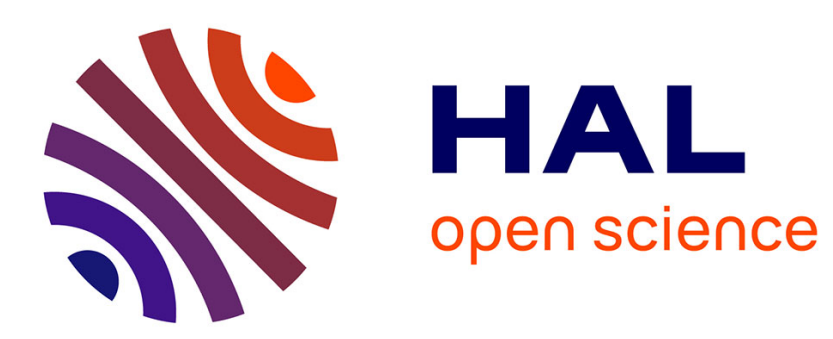

\title{
On Homogeneous Finite-Time Control for Linear Evolution Equation in Hilbert Space
}

\author{
Andrey Polyakov, Jean-Michel Coron, Lionel Rosier
}

\section{To cite this version:}

Andrey Polyakov, Jean-Michel Coron, Lionel Rosier. On Homogeneous Finite-Time Control for Linear Evolution Equation in Hilbert Space. IEEE Transactions on Automatic Control, 2018, 63 (9), pp.3143 - 3150. 10.1109/TAC.2018.2797838 . hal-01695475

\section{HAL Id: hal-01695475 \\ https://hal.inria.fr/hal-01695475}

Submitted on 29 Jan 2018

HAL is a multi-disciplinary open access archive for the deposit and dissemination of scientific research documents, whether they are published or not. The documents may come from teaching and research institutions in France or abroad, or from public or private research centers.
L'archive ouverte pluridisciplinaire HAL, est destinée au dépôt et à la diffusion de documents scientifiques de niveau recherche, publiés ou non, émanant des établissements d'enseignement et de recherche français ou étrangers, des laboratoires publics ou privés. 


\title{
On Homogeneous Finite-Time Control for Linear Evolution Equation in Hilbert Space
}

\author{
Andrey Polyakov, Jean-Michel Coron, Lionel Rosier
}

\begin{abstract}
Based on the notion of generalized homogeneity, a new algorithm of feedback control design is developed for a plant modeled by a linear evolution equation in a Hilbert space with a possibly unbounded operator. The designed control law steers any solution of the closed-loop system to zero in a finite time. Method of homogeneous extension is presented in order to make the developed control design principles to be applicable for evolution systems with non-homogeneous operators. The design scheme is demonstrated for heat equation with the control input distributed on the segment $[0,1]$.
\end{abstract}

\section{INTRODUCTION}

A certain type of symmetry of an operator with respect to the so-called dilation group is known as homogeneity [1], [2], [3], [4], [5], [6], [7]. Homogeneity simplifies qualitative analysis of nonlinear dynamical systems. For ordinary differential equations (ODEs) it allows local properties (e.g. local stability) to be extended globally, but asymptotic stability and negative homogeneity degree always imply finite-time stability [8], [9]. In order to ensure finite-time convergence control and/or observer design algorithm can be based on homogeneity [10], [11], [12], [13]. Homogeneous finite-time stabilization of linear plants is studied in [10], [14], [13], [15] but homogeneous finite-time observers are designed in [16], [17], [18]. Homogeneity allows robustness analysis to be done easily for essentially nonlinear systems [19], [20], [21].

Recently [22], a generalized group of dilations was introduced for Banach spaces allowing us to extend all important properties (known before only for ODEs) to homogeneous evolution equations in Banach and Hilbert spaces. The generalized homogeneity can be established for many wellknown partial differential equations (PDEs) like heat, wave, Korteweg-de Vries, Saint-Venant, Burgers, Navier-Stocks and fast diffusion equations [22].

The finite-time control of PDEs is still the topic of intensive research [23], [24], [25], [26], [27]. It is related with controllability analysis of evolution systems [28] as well as with sliding mode control method [29], [30].

This technical note deals with homogeneous finite-time control design for plants modeled by linear evolution equations in Hilbert spaces. The design scheme follows the idea of the implicit Lyapunov function method [31], [32], which was recently linked with homogeneity theory [33]. Using

This work is supported by ANR Project Finite4SoS (ANR 15-CE23-0007). Andrey Polyakov is with Inria Lille, 40. av Halley, Villenueve d'Ascq, France, (e-mail: andrey.polyakov@inria.fr) and also with ITMO University, SaintPetersburg, Kronverski av. 49, Russia. Jean-Michel Coron is with Laboratoire Jacques-Louis Lions, Université Pierre et Marie Curie, 4 place Jussieu, 75252 , Paris, France (e-mail: coron@ann.jussieu.fr). Lionel Rosier is with Centre Automatique et Systemes Ecole des Mines de Paris 60 Bd Saint-Miche, 75272, Paris,France, (e-mail: lionel.rosier@mines-paristech.fr). the monotone dilations, we introduce the so-called canonical homogeneous norm in a Banach space, which is utilized next for both control design and finite-time stability analysis. The preliminary version of this paper was presented at Conference on Decision and Control [34]. The key differences are as follows: the proof of the main theorem is presented; the results are extended to evolution equations with non-homogeneous operators which admit homogeneous (possibly set-valued) extensions; piecewise linear realization of the homogeneous feedback is studied; the new example of finite-time stabilization of heat system with Dirichlet boundary conditions on the segment $[0,1]$ is presented.

Notation. Set of reals (integers) is $\mathbb{R}\left(\mathbb{Z}\right.$ resp.); $\mathbb{R}_{+}=[0,+\infty) ;$ $H^{n}(\Omega, \mathbb{R})=W^{n, 2}(\Omega, \mathbb{R})$ is Sobolev space of functions $\Omega \rightarrow$ $\mathbb{R}, \Omega \subset \mathbb{R}$ is an open set; $C_{0}^{\infty}(\Omega, \mathbb{R})$ is the set of infinitely smooth functions having compact support in $\Omega ; H_{0}^{n}(\Omega, \mathbb{R})$ is the closure of $C_{0}^{\infty}(\Omega, \mathbb{R})$ with respect to the norm in $H^{n} ; \bar{X}$ denotes the closure of the set $X$ of a metric space.

\section{Problem Statement and Preliminaries}

Let us consider the following control system

$$
\begin{gathered}
\dot{u}(t)=A u(t)+B \xi(u(t)), \quad t>0 \\
u(0)=u_{0} \in \mathcal{D}(A),
\end{gathered}
$$

where $A: \mathcal{D}(A) \subset \mathbf{H} \rightarrow \mathbf{H}$ is a (possibly unbounded) closed linear operator with the domain $\mathcal{D}(A)$ dense in $\mathbf{H}, B: \mathbf{X} \rightarrow \mathbf{H}$ is a linear bounded operator, $\mathbf{X}$ is a real Banach space, $\mathbf{H}$ is a real Hilbert space with the inner product $\langle\cdot, \cdot\rangle, u(t)$ is the system state, $\xi: \mathbf{H} \rightarrow \mathbf{X}$ is a (locally or globally) bounded feedback control.

The control aim is to steer any solution of the closed-loop system to zero in a finite time. The control design procedure is assumed to be based on the generalized homogeneity [22].

\section{A. Homogeneous Dilations in Banach Spaces}

Let $\mathcal{L}(\mathbf{B})$ be the space of linear bounded operators $\mathbf{B} \rightarrow \mathbf{B}$, where $\mathbf{B}$ is a real Banach space with a norm $\|\cdot\|$.

Definition 1 ([22]): A map $\boldsymbol{d}: \mathbb{R} \rightarrow \mathcal{L}(\boldsymbol{B})$ is called dilation in $\boldsymbol{B}$ if it satisfies

- Group property: $\boldsymbol{d}(0)=I \in \mathcal{L}(\boldsymbol{B})$ and $\boldsymbol{d}(t+s)=$ $\boldsymbol{d}(t) \boldsymbol{d}(s)$ for $t, s \in \mathbb{R}$;

- Strong continuity property: the map $\boldsymbol{d}(\cdot) u: \mathbb{R} \rightarrow \boldsymbol{B}$ is continuous for any $u \in \boldsymbol{B}$;

- Limit property: $\lim _{s \rightarrow-\infty}\|\boldsymbol{d}(s) u\|=0$ and $\lim _{s \rightarrow+\infty}\|\boldsymbol{d}(s) u\|=\infty$ uniformly on $u \in S$, where $S$ is the unit sphere in $\boldsymbol{B}$.

We refer reader to [22], [34] for more details about homogeneous dilations and homogeneous evolution systems. 
The dilation $\mathbf{d}$ is strongly continuous group of linear bounded invertible operators defined by an infinitesimal generator $G_{\mathbf{d}}: \mathcal{D}\left(G_{\mathbf{d}}\right) \subset \mathbf{B} \rightarrow \mathbf{B}$ given by $G_{\mathbf{d}} u=\lim _{s \rightarrow 0} \frac{\mathbf{d}(s) u-u}{s}$ with the domain $\mathcal{D}\left(G_{\mathbf{d}}\right)=\left\{u \in \mathbf{B}: \exists \lim _{s \rightarrow 0} \frac{\mathbf{d}(s) u-u}{s}\right\}$ being the linear subspace dense in $\mathbf{B}$. The generator $G_{\mathbf{d}}$ is a closed linear operator satisfying the property [35, Ch.1, Theorem 2.4]:

for any $u \in \mathcal{D}\left(G_{\boldsymbol{d}}\right)$ and any $s \in \mathbb{R}$ one holds $\boldsymbol{d}(s) u \in$ $\mathcal{D}\left(G_{\boldsymbol{d}}\right)$ and the function $\boldsymbol{d}(\cdot) u: \mathbb{R} \rightarrow \mathcal{D}\left(G_{\boldsymbol{d}}\right)$ is continuously differentiable, $\frac{d}{d s} \boldsymbol{d}(s) u=G_{\boldsymbol{d}} \boldsymbol{d}(s) u=\boldsymbol{d}(s) G_{\boldsymbol{d}} u$.

For example, if $\mathbf{B}=H^{0}(\mathbb{R}, \mathbb{R})$ then the dilation can defined as $(\mathbf{d}(s) u)(x)=e^{s} u\left(e^{-s} x\right), s \in \mathbb{R}, u \in \mathbf{B}, x \in \mathbb{R}$ has the generator $\left(G_{\mathbf{d}} u\right)(x)=-x u^{\prime}(x)+u(x)$ with the domain $\mathcal{D}\left(G_{\mathbf{d}}\right)=\left\{u \in H^{0}(\mathbb{R}, \mathbb{R}):-x u^{\prime}+u \in H^{0}(\mathbb{R}, \mathbb{R})\right\}$.

Definition 2 ([22]): A nonempty set $\mathcal{D} \subseteq \boldsymbol{B}$ is said to be $\boldsymbol{d}$-homogeneous if $\mathcal{D}$ is invariant with respect to dilation $\boldsymbol{d}$.

The set $\mathcal{D}$ can also be called $\mathbf{d}$-homogeneous cone, since a point $z$ belongs to $\mathcal{D}$ together with the homogeneous curve $\{\mathbf{d}(s) z: s \in \mathbb{R}\}$. The d-homogeneous set $\mathcal{D}$ becomes the conventional positive cone in $\mathbf{B}$ if $\mathbf{d}(s)=e^{s} I \in \mathcal{L}(\mathbf{B})$.

The homogeneous sphere of the radius $r$ is given by

$$
S_{\mathbf{d}}(r)=\{u \in \mathbf{B}: \mathbf{d}(-\ln (r)) u \in S\}, \quad r>0 .
$$

One has $S_{\mathbf{d}}(r)=\mathbf{d}(\ln (r)) S_{\mathbf{d}}(1)$. For $g \in \mathcal{L}(\mathbf{B})$ let us denote

$$
\|g\|_{\mathcal{L}}^{\mathcal{D}}=\sup _{u \in S \cap \mathcal{D}}\|g(u)\| \text { and }\lfloor g\rfloor_{\mathcal{L}}^{\mathcal{D}}=\inf _{u \in S \cap \mathcal{D}}\|g(u)\| .
$$

Definition 3 ([34]): A dilation $\boldsymbol{d}$ is (strictly) monotone on $\mathcal{D} \subset \boldsymbol{B}$ if $\|\mathbf{d}(s)\|_{\mathcal{L}}^{\mathcal{D}}<1$ (resp. $\exists \beta>0:\|\mathbf{d}(s)\|_{\mathcal{L}}^{\mathcal{D}}<e^{\beta s}$ ), $\forall s<0$.

If the dilation $\mathbf{d}$ is monotone on $\mathcal{D} \subset \mathbf{B}$ then (see [34])

1. for any $u \in \mathcal{D} \backslash\{\boldsymbol{0}\}$ there exists the unique pair $\left(s_{0}, u_{0}\right) \in$ $\mathbb{R} \times S_{\mathbf{d}}(1)$ such that $u=\mathbf{d}\left(s_{0}\right) u_{0}$;

2. the function $\|\mathbf{d}(\cdot) u\|: \mathbb{R} \rightarrow \mathbb{R}_{+}$(with $u \in \mathcal{D} \backslash\{\boldsymbol{0}\}$ ) is continuous and strictly increasing on $\mathbb{R}$;

3. the function $\|\mathbf{d}(\cdot)\|_{\mathcal{L}}^{\mathcal{D}}: \mathbb{R} \rightarrow \mathbb{R}_{+}\left(\lfloor\mathbf{d}(\cdot)\rfloor_{\mathcal{L}}^{\mathcal{D}}: \mathbb{R} \rightarrow \mathbb{R}_{+}\right)$is lower (upper) semicontinuous and strictly increasing.

It is worth stressing that monotonicity of dilation depends on the norm $\|\cdot\|$. For example, the dilation $\boldsymbol{d}(s)=\left(\begin{array}{cc}e^{s} & 0 \\ 0 & e^{2 s}\end{array}\right)$ is monotone on $\boldsymbol{B}=\mathbb{R}^{2}$ equipped with the conventional Euclidean norm, but it is non-monotone on $\mathbb{R}^{2}$ equipped with the weighted norm $\|u\|_{P}=\sqrt{u^{T} P u}$ with $P=$ $\left(\begin{array}{cc}\frac{\sqrt{3}}{2} & \frac{1}{2} \\ -\frac{1}{2} & \frac{\sqrt{3}}{2}\end{array}\right)\left(\begin{array}{cc}100 & 0 \\ 0 & \frac{1}{100}\end{array}\right)\left(\begin{array}{cc}\frac{\sqrt{3}}{2} & -\frac{1}{2} \\ \frac{1}{2} & \frac{\sqrt{3}}{2}\end{array}\right)$. In the latter case, the curve $\{\boldsymbol{d}(s) u: s \in \mathbb{R}\}$ may cross the sphere $S$ in two different points.

Remark 1 (On dense set): If the dilation $\mathbf{d}$ is monotone on a set $\mathcal{D}$ dense in $\mathbf{B}$ then it is monotone on $\mathbf{B}$, since $\overline{\mathcal{D}}=\mathbf{B}$ and $\|\mathbf{d}(s)\|_{\mathcal{L}}^{\mathcal{D}}=\|\mathbf{d}(s)\|_{\mathcal{L}}^{\mathbf{B}}$.

Remark 2 (On a uniformly continuous dilation): Let the generator $G_{\mathbf{d}}$ be a bounded linear operator. Then the group d is uniformly continuous, $\|\mathbf{d}(s)\|_{\mathcal{L}}^{\mathbf{B}} \leq e^{s\left\|G_{\mathbf{d}}\right\|_{\mathcal{L}}^{\mathbf{B}}}$ for $s>0$ and $\frac{d}{d s} \mathbf{d}(s)=G_{\mathbf{d}} \mathbf{d}(s)=\mathbf{d}(s) G_{\mathbf{d}}$ for any $s \in \mathbb{R}$ (see [35, Ch.1]).

\section{B. Canonical homogeneous norm}

Let us introduce the so-called homogeneous norm in $\mathbf{B}$.

Definition 4: A continuous functional $p: \boldsymbol{B} \rightarrow \mathbb{R}_{+}$is said to be $\boldsymbol{d}$-homogeneous norm in $\boldsymbol{B}$ if $p(u) \rightarrow 0$ as $u \rightarrow \boldsymbol{O}$ and $p(\boldsymbol{d}(s) u)=e^{s} p(u)>0$ for $u \in \boldsymbol{B} \backslash\{\boldsymbol{\theta}\}, s \in \mathbb{R}$.
The functional $p$ may not satisfy triangle inequality $p(u+$ $v) \leq p(u)+p(v)$, so it is not a semi-norm. However, many authors (see e.g. [13], [36], [9]) call functionals satisfying the above definition by "homogeneous norm". We follow this tradition and consider the canonical homogeneous norm

$$
\|u\|_{\mathbf{d}}=e^{s_{u}}, \text { where } s_{u} \in \mathbb{R}:\left\|\mathbf{d}\left(-s_{u}\right) u\right\|=1 .
$$

defined for monotone dilation. Obviously, $\|\mathbf{d}(s) u\|_{\mathbf{d}}=e^{s}\|u\|_{\mathbf{d}}$.

Lemma 1: If the dilation $\boldsymbol{d}$ is monotone on $\boldsymbol{B}$ then the functional $\|\cdot\|_{\boldsymbol{d}}: \boldsymbol{B} \backslash\{\boldsymbol{0}\} \rightarrow \mathbb{R}_{+}$defined by (4) is single-valued and positive. It can be prolonged to zero by continuity since $\|u\|_{\boldsymbol{d}} \rightarrow 0$ as $\|u\| \rightarrow 0$. Moreover, if $\boldsymbol{d}$ is strictly monotone on $\boldsymbol{B}$ then $\|\cdot\|_{\boldsymbol{d}}$ is Lipschitz continuous on $\boldsymbol{B} \backslash\{\boldsymbol{0}\}$.

In [34] such a homogeneous norm was called canonical since it is induced by the canonical norm $\|\cdot\|$ in $\mathbf{B}$ and $\|u\|_{\mathbf{d}}=$ $1 \Leftrightarrow\|u\|=1$. Obviously, $\|u\|_{\mathbf{d}}=r$ implies $u \in S_{\mathbf{d}}(r), r \geq 0$ and for any $u \in \mathbf{B}$ we have

$$
\left\lfloor\mathbf{d}\left(\ln \left(\|u\|_{\mathbf{d}}\right)\right)\right\rfloor_{\mathcal{L}}^{\mathbf{B}} \leq\|u\| \leq\left\|\mathbf{d}\left(\ln \left(\|u\|_{\mathbf{d}}\right)\right)\right\|_{\mathcal{L}}^{\mathbf{B}}
$$

The canonical homogeneous norm $\|\cdot\|_{\mathbf{d}}$ is not a norm in a usual sense, but it defines a sort of topology in B. Indeed, the homogeneous sphere is given by (3) and the homogeneous ball can be defined as $B_{\mathbf{d}}(r)=\left\{u \in \mathbf{B}:\|u\|_{\mathbf{d}}<r\right\}$.

Lemma 2: Let $\boldsymbol{d}$ be a dilation in a Hilbert space $\boldsymbol{H}$ and $\exists \beta>0$ such that $\left\langle G_{\boldsymbol{d}} v, v\right\rangle \geq \beta\|v\|^{2}, v \in \mathcal{D} \subset \mathcal{D}\left(G_{\boldsymbol{d}}\right)$, where the set $\mathcal{D}$ is dense in $\boldsymbol{H}$. Then the dilation $\boldsymbol{d}$ is strictly monotone on $\boldsymbol{H}$, the homogeneous norm $\|\cdot\|_{d}$ is Fréchet differentiable on $\mathcal{D}\left(G_{\boldsymbol{d}}\right) \backslash\{\boldsymbol{0}\}$ and the Fréchet derivative of $\|\cdot\|_{\boldsymbol{d}}$ at $u \in$ $\mathcal{D}\left(G_{\boldsymbol{d}}\right) \backslash\{\boldsymbol{0}\}$ is given by

$$
\left(D\|u\|_{\boldsymbol{d}}\right)(\cdot)=\frac{\left\langle\boldsymbol{d}\left(-\ln \|u\|_{\boldsymbol{d}}\right) \cdot, \boldsymbol{d}\left(-\ln \|u\|_{\boldsymbol{d}}\right) u\right\rangle}{\left\langle G_{\boldsymbol{d}} \boldsymbol{d}\left(-\ln \|u\|_{\boldsymbol{d}}\right) u, \boldsymbol{d}\left(-\ln \|u\|_{\boldsymbol{d}}\right) u\right\rangle}\|u\|_{\boldsymbol{d}} .
$$

Proofs of all claims are given in Appendix.

\section{Homogeneous Operator}

Operators to be studied in this paper are introduced by

Definition 5 (Homogeneous operator): An operator $f$ : $\mathcal{D}(f) \subset \boldsymbol{B} \rightarrow \boldsymbol{B}$ is said to be $\boldsymbol{d}$-homogeneous of degree $\nu$ if $\mathcal{D}(f)$ is $\boldsymbol{d}$-homogeneous set and

$$
f(\mathbf{d}(s) u)=e^{\nu s} \mathbf{d}(s) f(u), \quad s \in \mathbb{R}, \quad u \in \mathcal{D}(f),
$$

where $\boldsymbol{d}$ is a dilation in $\boldsymbol{B}$ and $\nu \in \mathbb{R}$.

Any linear operator is always d-homogeneous of zero degree if $\mathbf{d}(s)=e^{s} I \in \mathcal{L}(\mathbf{B})$. However, in some cases linear operator may also have negative or positive homogeneity degree dependently of the dilation group. For example, the operator $A=\frac{\partial^{2}}{\partial x^{2}}: H^{2}(\mathbb{R}, \mathbb{R}) \subset H^{0}(\mathbb{R}, \mathbb{R}) \rightarrow H^{0}(\mathbb{R}, \mathbb{R})$ is d-homogeneous of degree $\nu \in \mathbb{R}$ provided that the dilation $\mathbf{d}$ is defined as follows $(\mathbf{d}(s) u)(x)=e^{\alpha s} u\left(e^{0.5 \nu s} x\right), s \in \mathbb{R}$, $u \in H^{0}(\mathbb{R}, \mathbb{R}), x \in \mathbb{R}$ and $\nu<4 \alpha$.

Remark 3 (On set-valued homogeneous operator):

Definition 5 is applicable to a set-valued operator $F: \mathcal{D}(F) \subset \mathbf{B} \rightrightarrows \mathbf{B}$ provided that the identity (7) is understood in the set-theoretic sense (as equality of sets). It remains meaningful even for unbounded sets (values of $F$ ) since $\mathbf{d}(s)$ is a linear bounded invertible operator on $\mathbf{B}$. In this paper we introduce the relaxed set-theoretic condition of homogeneity of the operator $F$ given by the inclusion

$$
e^{\nu s} \mathbf{d}(s) F(u) \subset F(\mathbf{d}(s) u), \quad s \in \mathbb{R}, \quad u \in \mathcal{D}(F) .
$$


Evolution equations with d-homogeneous operators were studied in [22] and [34]. Below we basically use the next property: if a uniformly asymptotically stable evolution system is $\boldsymbol{d}$ homogeneous with negative degree then it is finite-time stable.

\section{Homogeneous Extension}

Homogeneous extension allows some homogeneity-based methods to be applied for non-homogeneous systems.

Definition 6: (Homogeneous extension) A (possibly) setvalued d-homogeneous operator $F: \mathcal{D}(F) \subset \boldsymbol{B} \rightrightarrows \boldsymbol{B}$ is said to be $\boldsymbol{d}$-homogeneous extension of an operator $f: \mathcal{D}(f) \subset \boldsymbol{B} \rightarrow \boldsymbol{B}$ if $f(u) \subset F(u)$ for any $u \in \mathcal{D}(f) \subset \mathcal{D}(F)$.

Set-valued homogeneous extensions appear, for example, as a result of Filippov regularization procedure applied to discontinuous vector field [12], [14]. It also allow us to simplify robust stability analysis of uncertain non-linear affine system $s^{(n)}=a+b \xi, s \in \mathbb{R},|a|<C, 0<b_{\min }<b<b_{\max }$ provided that the extended differential inclusion $s^{(n)} \in[-C, C]+$ $\left[b_{\min }, b_{\max }\right] \xi$ is homogeneous, where $\xi=\xi\left(s, \dot{s}, \ldots, s^{(n-1)}\right)$ is a homogeneous feedback law [12], [14].

In this paper we deal with the homogeneous extension

$$
F(u)=\bigcup_{s \in \mathbb{R}: \mathbf{d}(s) u \in \mathcal{D}(f)}\left\{e^{-\nu s} \mathbf{d}(-s) f(\mathbf{d}(s) u)\right\},
$$

having the domain $\mathcal{D}(F)=\bigcup_{s \in \mathbb{R}} \mathbf{d}(s) \mathcal{D}(f)$. By construction the set-valued operator $F$ is $\mathbf{d}$-homogeneous of degree $\nu$. It satisfies (7) in the set-theoretic sense (see, Remark 3). Formally, such a set-valued homogeneous extension is defined for any fixed $\nu \in \mathbb{R}$. For example, if $f(u)=-(1+0.5 \sin (u))) u$ with $u \in \mathbb{R}$ then $F(u)=-u \cdot(0,+\infty)$ for $\nu \neq 0$ and $F(u)=-u \cdot\left(\frac{1}{2}, \frac{3}{2}\right)$ for $\nu=0$. The issue of an appropriate selection of the parameter $\nu$ can be solved for a concrete $f$.

Below we utilize the homogeneous extension for Lyapunov analysis of a well-posed non-homogeneous evolution system $\dot{u}(t)=f(u(t))$ having classical (or strong) solutions $u(t) \in$ $D(f) \subset \boldsymbol{H}$. Notice that in the latter case we do not need to prove existence of solution to the extended evolution inclusion $\dot{u} \in F(u)$ but we simply use the fact $f(u(t)) \subset F(u(t))$ in order to estimate time derivative of the Lyapunov-like functional $V: \mathcal{D}(f) \backslash\{\boldsymbol{0}\} \rightarrow \mathbb{R}_{+}$defined as $V(u)=\|u\|_{\mathbf{d}}$. Indeed, if $\mathcal{D}(f) \subset \mathcal{D}\left(G_{\mathbf{d}}\right)$ and $\exists \beta>0$ such that $\left\langle G_{\mathbf{d}} z, z\right\rangle \geq \beta\|z\|^{2}$ for $z \in \mathcal{D}\left(G_{\mathbf{d}}\right)$ (see, Lemma 2) then the formula (6) gives

$$
\begin{aligned}
\dot{V}(u) & =\left(D\|u\|_{\mathbf{d}}\right)(f(u))=\frac{\left\langle\mathbf{d}\left(-\ln \|u\|_{\mathbf{d}}\right) f(u), \mathbf{d}\left(-\ln \|u\|_{\mathbf{d}}\right) u\right\rangle}{\left\langle G_{\mathbf{d}} \mathbf{d}\left(-\ln \|u\|_{\mathbf{d}}\right) u, \mathbf{d}\left(-\ln \|u\| \|_{\mathbf{d}}\right) u\right\rangle}\|u\|_{\mathbf{d}} \\
& \leq \sup _{y \in F(u)} \frac{\left\langle\mathbf{d}\left(-\ln \|u\|_{\mathbf{d}} y, \mathbf{d}\left(-\ln \|u\|_{\mathbf{d}}\right) u\right\rangle\right.}{\left\langle G_{\mathbf{d}} \mathbf{d}\left(-\ln \|u\|_{\mathbf{d}}\right) u, \mathbf{d}\left(-\ln \|u\|_{\mathbf{d}}\right) u\right\rangle}\|u\|_{\mathbf{d}} .
\end{aligned}
$$

for $u \in \mathcal{D}(f) \backslash\{\boldsymbol{0}\}$. Since $F$ is $\mathbf{d}$-homogeneous operator then $e^{\nu s} \mathbf{d}(s) F(u) \subset F(\mathbf{d}(s) u)$ for any $s \in \mathbb{R}$ and

$$
\dot{V}(u) \leq\|u\|_{\mathbf{d}}^{1+\nu} \frac{\sup _{v \in F\left(\mathbf{d}\left(-\ln \|u\|_{\mathbf{d}}\right) u\right)}\left\langle v, \mathbf{d}\left(-\ln \|u\|_{\mathbf{d}}\right) u\right\rangle}{\left\langle G_{\mathbf{d}} \mathbf{d}\left(-\ln \|u\|_{\mathbf{d}}\right) u, \mathbf{d}\left(-\ln \|u\|_{\mathbf{d}}\right) u\right\rangle} .
$$

Using $\left\|\mathbf{d}\left(-\ln \|u\|_{\mathbf{d}}\right) u\right\|=1$ we conclude that the inequality

$$
\alpha\left\langle G_{\mathbf{d}} z, z\right\rangle+\sup _{v \in F(z)}\langle v, z\rangle \leq 0, \quad \forall z \in \mathcal{D}(f):\|z\|=1
$$

implies

$$
\dot{V}(u) \leq-\alpha V^{1+\nu}(u), \quad \alpha>0, \quad u \in \mathcal{D}(f) \backslash\{\mathbf{0}\} .
$$

In the latter case the functional $V$ decays along any solution $u(t) \in \mathcal{D}(f)$ and vanishes in a finite time provided that $\nu<0$.
The above considerations remain consistent for the case of unbounded sets $F(u), u \in \mathcal{D}(f)$. For example, if $f: \mathbb{R} \rightarrow \mathbb{R}$ is defined as $f(u)=-(1+|u|) \sqrt{|u|} \operatorname{sign}(|u|), u \in \mathbb{R}$ and $\mathbf{d}(s)=e^{s}, \nu=-1 / 2$, then the formula (9) gives the set-valued d-homogeneous extension with $F(u)=-\sqrt{|u|} \operatorname{sign}(u)$. $(1,+\infty)$. The differential equation $\dot{x}(t)=f(x(t))$, obviously, has continuous right-hand side so its solutions are well-defined (at least locally). For the uniform dilation $\mathbf{d}(s)=e^{s}$ one has $G_{\mathbf{d}}=1$ and $V(u)=\|u\|_{\mathbf{d}}=|u|$. Hence, from (10) and (11) we derive $\dot{V} \leq-V^{1 / 2}$ since $z^{2}+\sup _{y \in F(z)} y z \leq 0$ for all $z \in \mathbb{R}:|z|=1$. We refer reader to [15], [33] for more details about application of this scheme in the finite-dimensional case.

\section{Homogeneous Finite-time Control Design}

In [15], [33] finite-time control is designed for linear finitedimensional plants using implicit Lyapunov function method and homogeneity. First, a linear stabilizing feedback and a corresponding quadratic Lyapunov function are designed. Next, homogeneous dilation is applied in order to derive a finite-time stabilizing feedback and a corresponding implicit Lyapunov function. In this paper, we follow the same idea and use the norm $\|\cdot\|_{\mathbf{d}}$ defined by (4) as a Lyapunov function.

Theorem 1: If $A: \mathcal{D}(A) \subset \boldsymbol{H} \rightarrow \boldsymbol{H}$ is a generator of a strongly continuous semigroup on $\boldsymbol{H}, B: \boldsymbol{B} \rightarrow \boldsymbol{H}$ is a linear bounded operator and

A) $\boldsymbol{d}_{1}$ is a uniformly continuous group of linear bounded operators in $\boldsymbol{H}$ and $\gamma\|z\|^{2} \leq\left\langle G_{\boldsymbol{d}_{1}} z, z\right\rangle, \forall z \in \boldsymbol{H}$ with $\gamma \geq 0$,

B) $\boldsymbol{d}$ be a dilation on $\boldsymbol{H}$ such that $\beta\|z\|^{2} \leq\left\langle G_{\boldsymbol{d}} z, z\right\rangle$ for $z \in \mathcal{D}(A) \subset \mathcal{D}\left(G_{\boldsymbol{d}}\right)$ and $\beta>\left\|G_{\boldsymbol{d}_{1}}\right\|_{\mathcal{L}}^{\boldsymbol{H}}$,

C) $\exists K \in \mathcal{L}(\boldsymbol{H}, \boldsymbol{X})$ such that $B K(\mathcal{D}(A)) \subset \mathcal{D}(A)$ and

$$
\left\langle\left(A+B K+\alpha G_{\boldsymbol{d}}\right) z, z\right\rangle \leq 0, \quad \forall z \in \mathcal{D}(A),
$$

where $\alpha>0$ is a positive number,

D) the operator $A$ be d-homogeneous of degree $-\mu<0$ with $\mu \in\left(0, \beta-\left\|G_{\boldsymbol{d}_{1}}\right\|_{\mathcal{L}}^{\boldsymbol{H}}\right]$ and $\boldsymbol{d}(s) B K \boldsymbol{d}_{1}(s)=B K \boldsymbol{d}(s), s \in \mathbb{R}$, then the feedback control $\xi: \boldsymbol{H} \rightarrow \boldsymbol{H}$ defined by

$$
\xi(u)=\left\{\begin{array}{cl}
\|u\|_{\boldsymbol{d}}^{-\mu} K \boldsymbol{d}_{1}\left(-\ln \left(\|u\|_{\boldsymbol{d}}\right)\right) u & \text { if } u \neq 0, \\
0 & \text { if } u=0
\end{array}\right.
$$

is Lipschitz continuous on $\boldsymbol{H} \backslash\{\boldsymbol{0}\}$, Fréchet differentiable on $\mathcal{D}\left(G_{\boldsymbol{d}}\right) \backslash\{\boldsymbol{0}\}$ and $B \xi(\mathcal{D}(A)) \subset \mathcal{D}(A)$. If $\beta>\mu+\left\|G_{\boldsymbol{d}_{1}}\right\|_{\mathcal{L}}^{\boldsymbol{H}}$ then $\xi$ is continuous at $\boldsymbol{O} \in \boldsymbol{H}$. If $\exists M \geq 1$ such that $\|\boldsymbol{d}(s)\|_{\mathcal{L}}^{\boldsymbol{H}} \leq$ $M e^{(\mu+\gamma) s}$ for $s>0$ then $\|\xi(\cdot)\| \leq M\|K\|_{\mathcal{L}}$.

For any $u_{0} \in \mathcal{D}(A) \backslash\{\boldsymbol{0}\}$ the closed-loop system (1), (2), (12) has a unique classical solution ${ }^{1} u:\left[0, T\left(u_{0}\right)\right) \rightarrow \boldsymbol{H}$ such that $\|u(t)\| \rightarrow 0$ as $t \rightarrow T\left(u_{0}\right)$ and

$$
\frac{d}{d t}\|u(t)\|_{\mathbf{d}} \leq-\alpha\|u(t)\|_{\mathbf{d}}^{1-\mu} \quad t \in\left[0, T\left(u_{0}\right)\right),
$$

and the settling time admits the estimate $T\left(u_{0}\right) \leq \frac{\left\|u_{0}\right\|_{d}^{\mu}}{\alpha \mu}$.

The finite-time extinction of classical solutions obviously follows from the inequality (13), which can be derived using conditions of Theorem 1. Indeed, Condition D) asks for $\mathbf{d}$ homogeneity of the operator $f:=A+B \xi$ with negative degree $-\mu<0$, so one has $e^{-\mu s} \mathbf{d}(s) f(u)=f(\mathbf{d}(s) u)=$

${ }^{1}$ A continuous function $u:[0, T) \rightarrow \mathbf{H}$ is called classical solution to the initial value problem (1), (2) if it is continuously differentiable on $(0, T)$, $u(0)=u_{0}, u(t) \in \mathcal{D}(A)$ and $\dot{u}(t)=A u(t)+B \xi(u(t))$ for all $t \in(0, T)$. 
$(A+B K) \mathbf{d}(s) u$ for $s=-\ln \left(\|u\|_{\mathbf{d}}\right)$ and $u \in \mathcal{D}(A)$. Hence, Condition C) and the formula (6) yield (13). The detailed proof of Theorem 1 is given in Appendix. If Condition D) does not hold (non-homogeneous case) then the finite-time extinction can be analyzed using extension of $A$.

Corollary 1: Theorem 1 remains true if $D)$ is replaced with

$\left.\mathrm{D}^{*}\right)$ there exists a set-valued operator $F: \mathcal{D}(F) \subset \boldsymbol{H} \rightrightarrows \boldsymbol{H}$ such that $A u+B \xi(u) \in F(u)$ for $u \in \mathcal{D}(A) \subset \mathcal{D}(F)$, $F$ is $\boldsymbol{d}$-homogeneous of degree $-\mu<0$ and

$$
\alpha\left\langle G_{\boldsymbol{d}} z, z\right\rangle+\sup _{y \in F(z)}\langle y, z\rangle \leq 0, \quad z \in \mathcal{D}(F):\|z\|=1 .
$$

The operator $F$ is the homogeneous extension of the operator $A+B \xi$ (see Definition 6). Recall (see Section II-D) that we do not need to study an evolution system $\dot{u} \in F(u)$. The only fact $A u+B \xi(u) \in F(u)$ is utilized for analysis of finite-time extinction of the system (1). Notice that adding a linear feedback $\xi_{\text {new }}=K_{0} u+\xi, K_{0} \in \mathcal{L}(\mathbf{H}, \mathbf{X})$ we may allow the operator $A_{0}=A+B K_{0}$ to become homogeneous with a negative degree (see [33] for more details).

Corollary 2: If all conditions of Theorem 1 or Corollary 1 hold then for any $u_{0} \in \boldsymbol{H} \backslash\{\boldsymbol{0}\}$ the closed-loop system (1), (2), (12) has a unique mild solution $u:\left[0, T\left(u_{0}\right)\right) \rightarrow \boldsymbol{H}$ given by

$$
u(t)=\Phi(t) u_{0}+\int_{0}^{t} \Phi(t-s) B \xi(u(s)) d s,
$$

where $\{\Phi(t)\}_{t \geq 0}$ is a strongly continuous semigroup of linear bounded operators generated by the operator A. Moreover,

$$
\|u(t)\|_{\boldsymbol{d}} \leq\left\|u_{0}\right\|_{\boldsymbol{d}}-\alpha \int_{0}^{t}\|u(s)\|_{\boldsymbol{d}}^{1-\mu} d s, \quad t \in\left[0, T\left(u_{0}\right)\right)
$$

and $\|u(t)\| \rightarrow 0$ as $t \rightarrow T\left(u_{0}\right)$, where the settling time admits the estimate $T\left(u_{0}\right) \leq \frac{\left\|u_{0}\right\|_{d}^{\mu}}{\alpha \mu}$.

This corollary allows any mild solution of the closed-loop system to be prolonged to zero by continuity $u(t)=\mathbf{0}$ for $t \geq T\left(u_{0}\right)$ since the canonical homogeneous norm $\|\cdot\|_{\mathbf{d}}$ is the Lyapunov function to the closed-loop system implying uniqueness and finite-time stability of the zero solution.

Realization of the control law (12) needs calculation of the homogeneous norm $\|u\|_{\mathbf{d}}$, which is defined implicitly by the functional equation (4). Sometimes the homogeneous norm can be calculated explicitly (see, [15], [34] and the example below). The control law (12) can also be applied in a sampled way by means of replacing $\|u(t)\|_{\mathbf{d}}$ in (12) with $\left\|u\left(t_{j}\right)\right\|_{\mathbf{d}}$ for $t \in\left[t_{j}, t_{j+1}\right)$. The value $\left\|u\left(t_{j}\right)\right\|_{\mathbf{d}}$ can be found by means of an appropriate numerical procedure (see, e.g. [15]). The control (12) becomes linear on each interval of time $\left[t_{j}, t_{j+1}\right)$.

Let us consider the following switched linear feedback

$$
\xi_{\sigma(t)}(u(t))=r_{\sigma(t)}^{-\mu} K \mathbf{d}_{1}\left(-\ln \left(r_{\sigma(t)}\right)\right) u(t),
$$

with state dependent switching function $\sigma$ such that $\sigma(0) \in \mathbb{Z}$,

$$
\begin{gathered}
\sigma(t)=S\left(\sigma\left(t^{-}\right), u\left(t^{-}\right)\right), \quad S: \mathbb{Z} \times \mathbf{H} \rightarrow \mathbb{Z}, \\
S(\sigma, u):=\left\{\begin{array}{c}
i-1 \text { if } \sigma=i \text { and }\|u\|_{\mathbf{d}} \geq r_{i-1}, \\
i \quad \text { if } \sigma=i \text { and } r_{i+1} \leq\|u\|_{\mathbf{d}} \leq r_{i-1}, \\
i+1 \text { if } \sigma=i \text { and } r_{i+1} \leq\|u\|_{\mathbf{d}},
\end{array}\right.
\end{gathered}
$$

where $r_{i} \in \mathbb{R}_{+}: r_{i+1}<r_{i}, i \in \mathbb{Z}$ and $\Omega=\bigcup_{i \in \mathbb{Z}}\left\{r_{i}\right\}$. The switching variable $\sigma$ has its own evolution equation given by (17) so the pair $(u(t), \sigma(t))$ can be treated as an augmented state of the closed-loop switched system. We refer the reader to [37] for more details about switched systems.
Corollary 3 (On switched linear finite-time feedback law): If all conditions of Theorem 1 hold and the control is given by (16), (17) with $r_{0}=1, r_{i+1}=e^{-q_{i}} r_{i}, i \in \mathbb{Z}$ and $q_{i}>0$ such that $\sum_{i=0}^{+\infty} q_{i}=+\infty$ and $\lim _{i \rightarrow+\infty} q_{i+1} e^{-\mu q_{i}} / q_{i}<1$ then - for $u_{0} \in \mathcal{D}(A) \backslash\{\boldsymbol{0}\}$ the closed-loop system (1), (2), (16), (17) has a unique strong solution ${ }^{2}$ and $\|u(t)\| \rightarrow 0$ as $t \rightarrow T\left(u_{0}\right)$, where $T\left(u_{0}\right) \leq \alpha^{-1} \sum_{j=i_{0}}^{\infty} r_{j}^{\mu} q_{j}<+\infty$ if $\left\|u_{0}\right\|_{\boldsymbol{d}} \in\left(r_{i_{0}+1}, r_{i_{0}}\right]$; - the time instants $t_{j}:\left\|u\left(t_{j}\right)\right\|_{\boldsymbol{d}}=r_{j}$ are isolated and the set $\left\{t_{j}\right\}_{j=i_{0}+1}^{+\infty}$ has the unique accumulation point $T\left(u_{0}\right)$;

- the control is linear continuous feedback on each $\left(t_{j}, t_{j+1}\right)$ and $\left\|\xi_{\sigma(t)}(t)\right\| \leq M\|K\|_{\mathcal{L}}$ if $\|\boldsymbol{d}(s)\|_{\mathcal{L}}^{\boldsymbol{H}} \leq M e^{(\mu+\gamma) s}$ for $s>0$.

Existence, uniqueness and finite-time extinction of mild solutions to the closed-loop system (1), (2), (16), (17) with $u_{0} \in \mathbf{H} \backslash\{\boldsymbol{0}\}$ can be shown repeating the proof of Corollary 2 .

Corollary 3 proposes a scheme for homogeneity-based finite-time control design in the form of switched linear feedback. Its applicability to boundary control case looks like a promising research direction, especially, in the context of the papers [26], [38], where a finite-time linear switched boundary control has been designed for heat system using the backstepping approach [39].

\section{EXAmple: Distributed Finite-time CONTROL FOR HEAT SYSTEM}

Application of Theorem 1 for ODEs can be found in [33] and for PDEs defined on unbounded domains in [34], where, in particular, homogeneous finite-time feedback control is designed for wave equation. To demonstrate a possible way of application of Corollary 1, in this paper, we design a distributed finite-time control for heat system on $[0,1]$, namely, $\frac{\partial u}{\partial t}=\frac{\partial^{2} u}{\partial x^{2}}+\phi(x) \xi(t, x), u(t, 0)=u(t, 1)=0, u(0, x)=u_{0}(x)$, where $u$ is the state, $\xi$ is the distributed control, $\phi:[0,1] \rightarrow$ $\mathbb{R}_{+}$is a continuous function such that

$$
c x^{2} \leq \phi(x) \quad \text { for } \quad x \in[0,1],
$$

for some $c>0$. The function $\phi$ represents possibly nonuniform feedback gain on $[0,1]$. The problem of distributed finite-time control design for the heat system with $\phi(x)=1$ has been studied in [30]. Initially, we design a homogeneous finite-time control for a system virtually extended to $\mathbb{R}_{+}$. Next, we reduce the extended closed-loop system to the original one provided that the initial condition has a support only in $(0,1)$.

\section{A. Homogeneous finite-time control design on $\mathbb{R}_{+}$}

Let $\mathbf{H}=H^{0}\left(\mathbb{R}_{+}, \mathbb{R}\right)$ and the operator $A: \mathcal{D}(A) \subset \mathbf{H} \rightarrow \mathbf{H}$ be the connection of the Laplace operator with Dirichlet boundary conditions on $[0,1]$ and the zero operator on $[1,+\infty)$, i.e. $(A u)(x)=\frac{\partial^{2} u(x)}{\partial x^{2}}$ if $x \in(0,1)$ and $(A u)(x)=0$ if $x \geq 1$ for $u \in \mathcal{D}(A)=\left\{z \in \mathbf{H}: \begin{array}{c}z_{(0,1)} \in H_{0}^{1}((0,1), \mathbb{R}) \cap H^{2}((0,1), \mathbb{R}), \\ z_{(1,+\infty)} \in C_{0}^{\infty}((1,+\infty), \mathbb{R})\end{array}\right\}$, where $z_{(a, b)}$ denotes the restriction of $z$ to the interval $(a, b)$. Operator

\footnotetext{
${ }^{2} \mathrm{~A}$ continuous function $u:[0, T) \rightarrow \mathbf{H}$ which is differentiable almost everywhere on $[0, T]$ such that $\dot{u} \in L^{1}((0, T), \mathbf{H})$ is called strong solution to the initial value problem (1), (2), (16), (17) if $u(t) \in \mathcal{D}(A)$ for $t \in$ $(0, T), u(0)=u_{0}$ and there exists a (locally) piecewise constant function $\sigma:[0, T) \rightarrow \mathbb{Z}$ such that $\sigma$ satisfies (17) on $(0, T)$ and $\dot{u}(t)=A u(t)+$ $B \xi_{\sigma(t)}(u(t))$ almost everywhere on $(0, T)$.
} 
$A$ generates the strongly continuous semigroup $\{\Phi(t)\}_{t \geq 0}$ : $(\Phi(t) u)(x)=\left\{\begin{array}{cr}2 \sum_{n=1}^{\infty} e^{-n^{2} \pi^{2} t} \sin (\pi n x) \int_{0}^{1} \sin (\pi n y) u(y) d y & \text { if } x \in(0,1), \\ u(x) & \text { if } x \in[1,+\infty) .\end{array}\right.$

Let us consider the equation (1) with $A$ given above and $B$ : $\boldsymbol{H} \rightarrow \boldsymbol{H}$ defined as follows $(B z)(x)=\phi(x) z(x)$ if $x \in(0,1)$ and $(B z)(x)=\phi(1) z(x)$ if $x \geq 1$ for $z \in \boldsymbol{H}$. Let us show that conditions A)-C) of Theorem 1 and the condition $\left.D^{*}\right)$ of Corollary 1 hold for $K=-\rho I \in \mathcal{L}(\boldsymbol{H})$ with a sufficiently large scalar $\rho>0$.

A) Let the uniformly continuous dilation $\mathbf{d}_{1}$ be defined as $\left(\mathbf{d}_{1}(s) u\right)(x)=e^{\mu s} u(x)$ if $x \in(0,1)$ and $\left(\mathbf{d}_{1}(s) u\right)(x)=u(x)$ if $x \geq 1$. Hence, $0 \leq\left\langle G_{\mathbf{d}_{1}} u, u\right\rangle, u \in \mathbf{H}$ and $\left\|G_{\mathbf{d}_{1}}\right\|_{\mathcal{L}}^{\mathbf{H}}=\mu>0$.

B) Let the dilation $\mathbf{d}(s)$ in $\mathbf{H}$ be defined as $(\mathbf{d}(s) u)(x)=e^{s}$. $u\left(e^{-0.5 \mu s} x\right)$. It has the generator $G_{\mathbf{d}} u=u-0.5 \mu x u^{\prime}$ with the domain $\mathcal{D}\left(G_{\mathbf{d}}\right)=\left\{u \in \mathbf{H}: u-0.5 \mu x u^{\prime} \in \mathbf{H}\right\}$. Obviously,

$$
\|\mathbf{d}(s) u\|=\sqrt{\int_{\mathbb{R}_{+}} e^{2 s} u^{2}\left(e^{-0.5 \mu s} x\right) d x}=e^{(1+0.25 \mu) s}\|u\|
$$

and $(1+0.25 \mu)\|u\|^{2} \leq\left\langle G_{\mathbf{d}} u, u\right\rangle$ if $u \in \mathcal{D}(A) \subset \mathcal{D}\left(G_{\mathbf{d}}\right)$. Hence, we derive $\|u\|_{\mathbf{d}}=\|u\|^{\frac{1}{1+0.25 \mu}}$ and the formula (12) gives $\xi(u)=-\rho\|u\|_{\mathbf{d}}^{-\mu} B \mathbf{d}_{1}\left(-\ln \|u\|_{\mathbf{d}}\right) u$.

According to Theorem 1 this feedback control $\xi$ is locally bounded and continuous on $\mathbf{H}$ if $0<\mu<4 / 7$ and globally bounded if $\mu=4 / 7$.

C)-D*) Let us consider the homogeneous extension (9), i.e.

$$
\left.F(u)=\bigcup_{s \in \mathbb{R}: \mathbf{d}(s) u \in \mathcal{D}(A)}\left\{A_{s} u+q_{s}(u)\right)\right\},
$$

where $A_{s}=e^{\mu s} \mathbf{d}(-s) A \mathbf{d}(s)$ and $q_{s}(u)=e^{\mu s} \mathbf{d}(-s) B \xi(\mathbf{d}(s) u)$. It is easy to see that $\left(A_{s} u\right)(x)=u^{\prime \prime}(x)$ if $x \in\left(0, e^{-0.5 \mu s}\right)$ and $\left(A_{s} u\right)(x)=0$ if $x \geq e^{-0.5 \mu s}$,

$$
\left(q_{s}(u)\right)(x)=-\rho\left\{\begin{array}{cc}
\phi\left(e^{0.5 \mu s} x\right) e^{-\mu s} \frac{u(x)}{\|u\|_{\mathbf{d}}^{2 \mu}}, & x \in\left(0, e^{-0.5 \mu s}\right), \\
\phi(1) \frac{u(x)}{\|u\|_{\mathbf{d}}^{\mu}}, & x \geq e^{-0.5 \mu s} .
\end{array}\right.
$$

The set-valued operator $F: \mathcal{D}(F) \subset \boldsymbol{H} \rightrightarrows \boldsymbol{H}$ is $\boldsymbol{d}$ homogeneous of degree $-\mu<0$ and $A u+B \xi(u) \in F(u)$ for $u \in \mathcal{D}(A)$, where $\mathcal{D}(F)=\bigcup_{s \in \mathbb{R}} \boldsymbol{d}(s) \mathcal{D}(A)$.

Let us denote $\langle u, v\rangle_{(a, b)}=\int_{a}^{b} u(x) v(x) d x$. For $\mathbf{d}(s) z \in \mathcal{D}(A)$ and $q_{s}=e^{-0.5 \mu s}$ using integration by parts we derive $\left\langle x z^{\prime}, z\right\rangle_{\left(0, q_{s}\right)}=\frac{-\langle z, z\rangle_{\left(0, q_{s}\right)}}{2}$ and $\left\langle x z^{\prime}, z\right\rangle_{\left(q_{s},+\infty\right)}=\frac{-\langle z, z\rangle_{\left(q_{s},+\infty\right)}}{2}$. Due to (18) one has $\phi\left(e^{0.5 \mu s} x\right) e^{-\mu s} \geq c x^{2}$ if $x \in\left[0, e^{-0.5 \mu s}\right]$ and $\left\langle\phi\left(e^{0.5 \mu s} x\right) e^{-\mu s} z, z\right\rangle_{\left(0, e^{-0.5 \mu s}\right)} \geq c\langle x z, x z\rangle_{\left(0, e^{-0.5 \mu s}\right)}$. Since $G_{\mathbf{d}} z=z-\mu x z^{\prime}$ then for $\|z\|=1$ we obtain

$$
\begin{gathered}
\left\langle\left(A_{s} z+q_{s}(z)+\alpha G_{\mathbf{d}}\right) z, z\right\rangle \leq-\left\langle z^{\prime}, z^{\prime}\right\rangle_{\left(0, q_{s}\right)} \\
-2 \alpha(1+0.25 \mu)\left\langle x z^{\prime}, z\right\rangle_{\left(0, q_{s}\right)}-\rho c\langle x z, x z\rangle_{\left(0, q_{s}\right)} \\
-(\rho \phi(1)-\alpha(1+0.25 \mu))\langle z, z\rangle_{\left(q_{s},+\infty\right)} \leq 0
\end{gathered}
$$

provided that $\rho \geq \max \left\{\frac{\alpha(1+0.25 \mu)}{\phi(1)}, \frac{\left.\alpha^{2}(1+0.25 \mu)^{2}\right)}{c}\right\}$.

Taking into account $A_{0}=A, q_{0}=B \xi$ we conclude that the conditions $A)-C$ ) of Theorem 1 and the condition $\mathrm{D}^{*}$ ) of Corollary 1 hold, so the feedback law (12) steers any solution of (1) to zero in a finite time $T \leq(\alpha \mu)^{-1}\left\|u_{0}\right\|_{\mathbf{d}}^{\mu}$.

Remark 4: Note that all constructions presented above remains correct if on the left boundary we have the homogeneous Neumann boundary condition: $\frac{\partial u}{\partial x}(t, 0)=0$. Extension of the proposed methodology to other types of boundary conditions is an interesting problem of future research.

\section{B. Restriction of finite-time control to the segment $[0,1]$}

If $\operatorname{supp}(u) \subset[0,1]$ and $u \in \mathbf{H} \backslash\{\mathbf{0}\}$ then $\operatorname{supp}(\Phi(t) u) \subset$ $[0,1], \operatorname{supp}(\xi(u)) \subset[0,1]$ and $\|u\|=\sqrt{\int_{0}^{1} u^{2}(x) d x}$, where $\operatorname{supp}[z]$ denotes the support of a function $z \in \mathcal{D}(A)$. Therefore, any classical solution of the closed-loop system (1), (12) with initial condition $u_{0} \in\{z \in \mathcal{D}(A): \operatorname{supp}(z) \subset[0,1]\}$ coincides on $[0,1]$ with the classical solution to the equation

$$
\frac{\partial u}{\partial t}=\frac{\partial^{2} u}{\partial x^{2}}+\phi(x) \frac{-\rho}{\|u\|^{\frac{2 \mu}{1+0.25 \mu}}} u, \quad \begin{gathered}
u(t, 0)=u(t, 1)=0, \\
u(0, x)=u_{0}(x), x \in[0,1] .
\end{gathered}
$$

Consequently, any solution of the latter heat system vanishes in a finite time $T\left(u_{0}\right) \leq(\alpha \mu)^{-1}\left(\int_{0}^{1} u_{0}^{2}(x) d x\right)^{0.5 \mu /(1+0.25 \mu)}$.

\section{Piecewise linear finite-time control on $[0,1]$}

Applying Corollary 3 for $q_{i}=\ln 2$ we derive $\xi(t)=$ $-\lambda(t) u(t)$, where the switched gain $\lambda(t)=\rho / r_{\sigma(t)}^{2 \mu}$ with $r_{i}=2^{-i}$ and $\sigma(t)$ defined by (17). Therefore, for any $u_{0} \in H_{0}^{1}((0,1), \mathbb{R}) \cap H^{2}((0,1), \mathbb{R})$ the equation

$$
\frac{\partial u}{\partial t}=\frac{\partial^{2} u}{\partial x^{2}}-\lambda(t) \phi(x) u, \quad \begin{gathered}
u(t, 0)=u(t, 1)=0, \\
u(0, x)=u_{0}(x), x \in[0,1] .
\end{gathered}
$$

has a unique strong solution which vanishes in a finite-time $T\left(u_{0}\right) \leq \frac{\ln 2}{\alpha} \sum_{j=i_{0}}^{+\infty} 2^{-\mu j}$ for $\left\|u_{0}\right\|_{\mathbf{d}} \in\left(r_{i_{0}+1}, r_{i_{0}}\right]$. Notice that in contrast to the linear switched feedback presented in [26], the settling time of our algorithm depends on the initial condition.

The simulation results of the system (19) (for $\phi(x)=x^{2}$ ) with homogeneous and switched linear finite-time controls $(\mu=0.5, \rho=1)$ as well as with the static linear feedback $(\lambda=\rho)$ are depicted at Fig. 1. The initial condition is given by $u_{0}(x)=5.25 x(1-x)$. For numerical simulations the system has been discretized by means of divided differences on a uniform grid with the step $h=0.005$ for the space variable. The discretization with respect to time has been done using implicit scheme with the step size $\tau=h^{2}$. The norm $\|u\|$ is calculated by Simpson rule. Numerical simulations confirm that the heat system with homogeneous control has fast decay rate (typical for finite-time stable system) with the settling time $T\left(u_{0}\right) \approx 0.4$.

\section{CONCLUSIONS AND Discussions}

The universal scheme of finite-time control design for a plant modeled by an evolution equation in a Hilbert space with a linear (possibly unbounded) operator is developed. The design procedure is essentially based on the concept of generalized homogeneity of operators in Banach/Hilbert spaces. The developed framework looks promising for extension of other homogeneity-based results (e.g. robustness (input-tostate stability) analysis [21] or finite/fixed-time observer design [16], [18]) to evaluation equations in Hilbert spaces.

\section{APPENDIX}

\section{A. Proof of Lemma 1}

Since the dilation is monotone $\mathbf{B}$ then for any $u \in \mathbf{B} \backslash\{\mathbf{0}\}$ there exists a unique pair $s_{0} \in \mathbb{R}$ and $u_{0} \in S$ such that $u=\mathbf{d}\left(s_{0}\right) u_{0}$ (see, Property 1 on the page 2 ). This means that $\left\|\mathbf{d}\left(-s_{0}\right) u\right\|=1$, i.e. $\|u\|_{\mathbf{d}}=e^{s_{0}}$ is finite, positive and uniquely defined for any $u \in \mathbf{B} \backslash\{\boldsymbol{0}\}$. Since $\left\|\mathbf{d}\left(-\ln \|u\|_{\mathbf{d}}\right) u\right\|=1$ for 


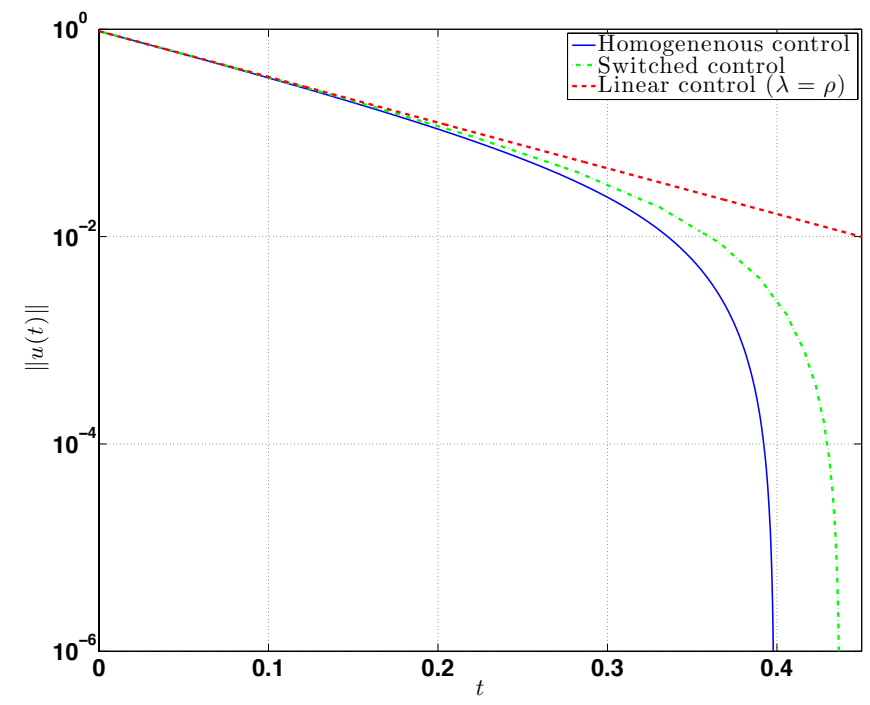

Fig. 1. Evolution of $L^{2}$-norm of the heat equation with linear, switched linear and homogeneous control laws plotted in the logarithmic scale

$u \in \mathbf{B} \backslash\{\mathbf{0}\}$ then $\left\|\mathbf{d}\left(-\ln \|u\|_{\mathbf{d}}\right)\right\|_{\mathcal{L}}^{\mathbf{B}} \geq \frac{1}{\|u\|}$. This means that $\left\|\mathbf{d}\left(-\ln \|u\|_{\mathbf{d}}\right)\right\|_{\mathcal{L}}^{\mathbf{B}} \rightarrow+\infty$ as $\|u\| \rightarrow 0$. Property 3 on the page 2 implies $\|u\|_{\mathbf{d}} \rightarrow 0$ as $\|u\| \rightarrow 0$. By definition of the homogeneous norm one has ||$u\left\|_{\mathbf{d}}^{\beta}-\right\| v \|_{\mathbf{d}}^{\beta}|=| e^{\beta s_{u}}-e^{\beta s_{v}} \mid$, where $\left\|\mathbf{d}\left(-s_{u}\right) u\right\|=1$ and $\left\|\mathbf{d}\left(-s_{v}\right) v\right\|=1$. On the one hand, for $s_{u}<s_{v}$ we have $1=\left\|\mathbf{d}\left(-s_{v}\right)(v-u)+\mathbf{d}\left(s_{u}-s_{v}\right) \mathbf{d}\left(-s_{u}\right) u\right\| \leq$ $\left\|\mathbf{d}\left(-s_{v}\right)\right\|_{\mathcal{L}}^{\mathbf{B}}\|u-v\|+\left\|\mathbf{d}\left(s_{u}-s_{v}\right)\right\|_{\mathcal{L}}^{\mathbf{B}} \leq\left\|\mathbf{d}\left(-s_{v}\right)\right\|_{\mathcal{L}}^{\mathbf{B}} \cdot\|u-v\|+$ $e^{\beta\left(s_{u}-s_{v}\right)}$. On the other hand, for $s_{u}>s_{v}$ we derive similarly $1 \leq\left\|\mathbf{d}\left(-s_{u}\right)\right\|_{\mathcal{L}}^{\mathbf{B}} \cdot\|u-v\|+e^{\beta\left(s_{v}-s_{u}\right)}$. Hence, if $\|u\|_{\mathbf{d}}>r$ and $\|v\|_{\mathbf{d}}>r$ then $e^{\beta s_{u}}\left\|\mathbf{d}\left(-s_{u}\right)\right\|_{\mathcal{L}}^{\mathbf{B}} \leq L, e^{\beta s_{v}}\left\|\mathbf{d}\left(-s_{v}\right)\right\|_{\mathcal{L}}^{\mathbf{B}} \leq L$ and $\left|\|u\|_{\mathbf{d}}^{\beta}-\|v\|_{\mathbf{d}}^{\beta}\right|=\left|e^{\beta s_{u}}-e^{\beta s_{v}}\right| \leq L\|u-v\|$, where $L=\max \left\{1,\|\mathbf{d}(-\ln r)\|_{\mathcal{L}}^{\mathbf{B}}\right\}$. The latter means that $\|\cdot\|_{\mathbf{d}}$ satisfies Lipschitz condition on any set $\mathbf{B} \backslash B_{\mathbf{d}}(r), r>0$.

\section{B. Proof of Lemma 2}

Since for any $u \in \mathcal{D} \subset \mathcal{D}\left(G_{\mathbf{d}}\right)$ we have $\frac{d}{d s}\|\mathbf{d}(s) u\|^{2}=$ $\frac{d}{d s}\langle\mathbf{d}(s) u, \mathbf{d}(s) u\rangle=2\left\langle G_{\mathbf{d}} \mathbf{d}(s) u, \mathbf{d}(s) u\right\rangle \geq \beta\|\mathbf{d}(s) u\|^{2}$ then $\left\|\mathbf{d}\left(s_{2}\right) u\right\| \geq e^{\beta\left(s_{2}-s_{1}\right)}\left\|\mathbf{d}\left(s_{1}\right) u\right\|$ for any $s_{1}<s_{2}$. Hence for $s_{2}=0$ and $s_{1}=s<0$ we have $\|\mathbf{d}(s) u\| \leq e^{\beta s}\|u\|$ for any $u \in \mathcal{D}$ and $\|\mathbf{d}(s)\|_{\mathcal{L}}^{\mathcal{D}}=\sup _{u \in \mathcal{D}} \frac{\|\mathbf{d}(s) u\|}{\|u\|} \leq e^{\beta s}$ for any $s \leq 0$. Since $\mathcal{D}$ is dense in $\mathbf{H}$ then $\overline{\mathcal{D}}=\mathbf{H}$ and $\|\mathbf{d}(s)\|_{\mathcal{L}}^{\mathcal{D}}=\|\mathbf{d}(s)\|_{\mathcal{L}}^{\mathbf{H}}$.

Since $\langle\mathbf{d}(-s) u, \mathbf{d}(-s) u\rangle=1$ for $\|u\|_{\mathbf{d}}=e^{s}$ then formula (6) follows from the implicit function theorem [40, Theorem E], the identity $(D\|u\|)(\cdot)=\frac{\langle\cdot, u\rangle}{\|u\|}$ and the identity $\frac{d}{d s} \mathbf{d}(s) u=$ $G_{\mathbf{d}} \mathbf{d}(s) u$, which holds for $u \in \mathcal{D}\left(G_{\mathbf{d}}\right)$.

\section{Proof of Theorem 1}

I. Properties of Control. Since the domain $\mathcal{D}(A)$ is dense in $\mathbf{H}$ then in the view of Lemmas 1 and 2 the inclusion $\mathcal{D}(A) \subset$ $\mathcal{D}\left(G_{\mathbf{d}}\right)$ and the first inequality in the condition C) imply that $\mathbf{d}$ is strictly monotone on $\mathbf{H},\|\mathbf{d}(s)\|_{\mathcal{L}}^{\mathbf{H}} \leq e^{\beta s}$ for $s \leq 0$, the homogeneous norm $\|\cdot\|_{\mathbf{d}}$ is continuous at $\mathbf{0}$, Lipschitz continuous on $\mathbf{H} \backslash\{\boldsymbol{0}\}$, Fréchet differentiable on $\mathcal{D}\left(G_{\mathbf{d}}\right) \backslash\{\boldsymbol{0}\}$ and the derivative of the homogeneous norm $\|\cdot\|_{\mathbf{d}}$ can be calculated by the formula (6). Similarly, we conclude that $\left\|\mathbf{d}_{1}(s)\right\|_{\mathcal{L}}^{\mathbf{H}} \leq e^{\gamma s}$ for $s \leq 0$. Taking into account $G_{\mathbf{d}_{1}} \in \mathcal{L}(\mathbf{H})$ we derive that $\xi$ is Fréchet differentiable on $\mathcal{D}\left(G_{\mathbf{d}}\right) \backslash\{\boldsymbol{0}\}$. Due to (5) we have $\|\xi(u)\| \leq\|u\|_{\mathbf{d}}^{-\mu}\|K\|_{\mathcal{L}}\left\|\mathbf{d}_{1}\left(-\ln \|u\|_{\mathbf{d}}\right)\right\|_{\mathcal{L}}^{\mathbf{H}}\left\|\mathbf{d}\left(\ln \|u\|_{\mathbf{d}}\right)\right\|_{\mathcal{L}}^{\mathbf{H}}$.
Hence, for $\|u\| \geq 1$ one has $\|\xi(u)\| \leq M\|K\|_{\mathcal{L}}$ if $\left\|\mathbf{d}\left(\ln \|u\|_{\mathbf{d}}\right)\right\|_{\mathcal{L}}^{\mathbf{H}} \leq M\|u\|_{\mathbf{d}}^{\mu+\gamma}$, but for $\|u\| \leq 1$ we derive $\|\xi(u)\| \leq\|u\|_{\mathbf{d}}^{\beta-\mu-\left\|G_{\mathbf{d}_{1}}\right\|_{\mathcal{L}}^{\mathbf{H}}}$, since $\left\|\mathbf{d}_{1}(s)\right\|_{\mathcal{L}}^{\mathbf{H}} \leq e^{s\left\|G_{\mathbf{d}_{1}}\right\|_{\mathcal{L}}^{\mathbf{H}}}$ if $s \geq 0$.

II. Existence of Solutions. Let us prove that for any initial condition $u(0)=u^{*} \in D(A) \backslash\{\boldsymbol{0}\}$ the system (1), (12) has a classical solution $u(t) \in \mathcal{D}(A)$ defined at least locally.

a) Let function $\tilde{\xi}:(0,+\infty) \times \mathbf{H} \rightarrow \mathbf{H}$ be defined as

$$
\tilde{\xi}(q, u)=q^{-\mu} B K \mathbf{d}_{1}(-\ln (q)) u .
$$

Since the group $\mathbf{d}_{1}$ is uniformly continuous and $\frac{d}{d s} \mathbf{d}_{1}(s)=$ $G_{\mathbf{d}_{1}} \mathbf{d}_{1}(s)$ for all $s \in \mathbb{R}$ then the function $\tilde{\xi}$ is continuously Fréchet differentiable on $(0,+\infty) \times \mathbf{H}$.

Let $T^{*}$ be an arbitrary positive real number and let $g$ : $\left[0, T^{*}\right] \rightarrow \mathbb{R}$ be an arbitrary continuous function such that $g(t)>0$ for all $t \in\left[0, T^{*}\right]$. To prove that Cauchy problem

$$
\begin{aligned}
& \dot{u}(t)=\hat{A}(g(t)) u(t):=A u(t)+\tilde{\xi}(g(t), u(t)), \\
& u(0)=u^{*} \in \mathcal{D}(A)
\end{aligned}
$$

has a unique classical solution $u_{g}(t) \in \mathcal{D}(A), t \in\left[0, T^{*}\right]$, we use [35, Chapter 5, Theorem 3.1], which needs to check the following three conditions:

H1) $\hat{A}(g(t))_{\left\{t \in\left[0, T^{*}\right]\right\}}$ is stable family of generators;

H2) $\mathbf{Y}$ is $\hat{A}(g(t))$-admissible, where $\mathbf{Y}=\mathcal{D}(A)$ is a Banach space with the graph norm $\|u\|_{\mathbf{Y}}=\|u\|+\|A u\|$;

H3) $\hat{A}(g(t)) \in \tilde{\mathcal{L}}:=\mathcal{L}(\mathbf{Y}, \mathbf{H})$ for $t \in\left[0, T^{*}\right]$ and $\hat{A}(g(\cdot))$ is continuous on $\left[0, T^{*}\right]$ in the norm $\|\cdot\|_{\tilde{\mathcal{L}}}=\sup _{y \in \mathbf{Y} \backslash\{0\}} \frac{\|\cdot y\|}{\|y\|+\|A y\|}$.

Since the function $g$ is continuous and positive on $\left[0, T^{*}\right]$ then $\tilde{\xi}(g(t), \cdot) \in \mathcal{L}(\mathbf{H})$, the conditions H1) and H2) hold and $\hat{A}(g(t)) \in \mathcal{L}(\mathbf{Y}, \mathbf{H})$ for all $t \in\left[0, T^{*}\right]$. Finally, the inequalities $\mid\|\hat{A}(g(t+\delta))\|_{\tilde{\mathcal{L}}}-\|\hat{A}(g(t))\|_{\tilde{\mathcal{L}}} \leq\|\hat{A}(g(t+\delta))-\hat{A}(g(t))\|_{\tilde{\mathcal{L}}}$

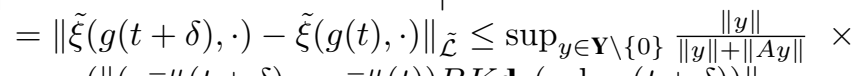
$\left(\left\|\left(g^{-\mu}(t+\delta)-g^{-\mu}(t)\right) B K \mathbf{d}_{1}(-\ln g(t+\delta))\right\|_{\mathcal{L}}\right.$

$\left.+\left\|g^{-\mu}(t) B K\left(\mathbf{d}_{1}(-\ln g(t+\delta))-\mathbf{d}_{1}(-\ln g(t))\right)\right\|_{\mathcal{L}}\right)$ and uniform continuity of the group $\mathbf{d}_{1}$ yield that condition H3) also holds. Therefore, the considered Cauchy problem (20) has the unique classical solution $u_{g}(t)$ on $\left[0, T^{*}\right]$.

b) Let the numbers $q_{\min }, q_{\max }, r_{\min }, r_{\max }$ be defined as follows: $0<q_{\min }<\inf \left\{\|u\|_{\mathbf{d}}:\|u\|=\left\|u^{*}\right\|\right\}, \sup \left\{\|u\|_{\mathbf{d}}:\|u\|=\right.$ $\left.\left\|u^{*}\right\|\right\}<q_{\max }<+\infty, r_{\min }=\sup \left\{\|u\| /\left\|u^{*}\right\|:\|u\|_{\mathbf{d}}=q_{\min }\right\}$, $r_{\max }=\inf \left\{\|u\| /\left\|u^{*}\right\|:\|u\|_{\mathbf{d}}=q_{\max }\right\}$. From definition of homogeneous norm we have that $r_{\min } \rightarrow 0$ as $q_{\min } \rightarrow 0$ and $r_{\max } \rightarrow+\infty$ as $q_{\max } \rightarrow+\infty$. Let us select $q_{\min }$ and $q_{\max }$ such that $r_{\min }<1<r_{\max }$. Let $\{\Phi(t)\}_{t>0}$ be strongly continuous semigroup generated by $A$ then there exist $\kappa>0$ and $R>0$ such that $\|\Phi(t) u\| \leq R e^{\kappa t}\|u\|$ for any $u \in \mathbf{H}$.

Let us consider the family of Cauchy problems

$$
\begin{aligned}
& \dot{u}_{n}(t)=A u_{n}(t)+\tilde{\xi}\left(q_{n-1}(t), u_{n}(t)\right), \quad t \in\left(0, T_{n-1}\right), \\
& u_{n}(0)=u^{*} \in \mathcal{D}(A) \backslash\{0\},
\end{aligned}
$$

where $n \geq 1, q_{n-1}$ and $T_{n}$ are defined recursively as follows $T_{0}>0: \frac{2\left\|\Phi(t) u^{*}\right\|-\left\|u^{*}\right\|}{\left\|u^{*}\right\|} \in\left[r_{\min }, r_{\max }\right], \forall t \in\left[0, T_{0}\right]$, $T_{n}=T_{n-1}$ if $\left\|u_{n}(\tau)\right\| \in\left(r_{\min }, r_{\max }\right), \forall \tau \in\left[0, T_{n-1}\right)$, $T_{n}=\inf \left\{\tau \in\left[0, T_{n-1}\right):\left\|u_{n}(\tau)\right\| \in\left\{r_{\min }, r_{\max }\right\}\right\}$ otherwise, $q_{0}(t)=\left\|u^{*}\right\|_{\mathbf{d}} \quad$ and $\quad q_{n}(t)=\left\|u_{n}(t)\right\|_{\mathbf{d}}$.

Since $q_{n-1}$ is a continuous positive function then the Cauchy problem (21) has unique classical solution $u_{n}(t) \in \mathcal{D}(A)$ on $\left[0, T_{n}\right]$. So the sequences $\left\{q_{n}\right\}$ and $\left\{u_{n}\right\}$ are well defined and $q_{\text {min }} \leq q_{n}(t) \leq q_{\max }, r_{\min }\left\|u^{*}\right\| \leq\left\|u_{n}(t)\right\| \leq r_{\max }\left\|u^{*}\right\|$. 
c) Let us show that $T^{*}:=\inf _{n} T_{n}>0$. Initially, note that $\left\|\tilde{\xi}\left(q_{n-1}(s), u_{n}(s)\right)\right\|=\left\|q_{n-1}^{-\mu}(s) B K \mathbf{d}\left(-\ln q_{n-1}(s)\right) u_{n}(s)\right\| \leq$ $\|B K\|_{\mathcal{L}}^{\mathbf{H}} q_{n-1}^{-\mu-\left\|G_{\mathbf{d}_{1}}\right\|_{\mathcal{L}}^{\mathbf{H}}}(s)\left\|u_{n}(s)\right\| \leq c\left\|u^{*}\right\|$, where $c=\|B K\|_{\mathcal{L}}^{\mathbf{H}}$. $\max \left\{q_{\max }^{-\mu-\left\|G_{\mathbf{d}_{1}}\right\|_{\mathcal{L}}^{\mathbf{H}}}, q_{\min }^{-\mu-\left\|G_{\mathbf{d}_{1}}\right\|_{\mathcal{L}}^{\mathbf{H}}}\right\} r_{\max }$. Hence, we derive $\frac{r_{\min }+1}{2}\left\|u^{*}\right\| \leq\left\|\Phi(t) u^{*}\right\| \leq\left\|u_{n}(t)\right\|+\left\|u_{n}(t)-\Phi(t) u^{*}\right\| \leq$ $\left\|u_{n}(t)\right\|+\int_{0}^{t}\left\|\Phi(t-s) \tilde{\xi}\left(q_{n-1}(s), u_{n}(s)\right)\right\| d s \leq\left\|u_{n}(t)\right\|+$ $c R\left\|u^{*}\right\| \int_{0}^{t} e^{\kappa(t-s)} d s \leq\left\|u_{n}(t)\right\|+\frac{c R\left\|u^{*}\right\|\left(e^{\kappa t}-1\right)}{\kappa}$. On the other hand, $\left\|u_{n}(t)\right\|=\left\|\Phi(t) u^{*}+\int_{0}^{t} \Phi(t-s) \tilde{\xi}\left(q_{n-1}(s), u_{n}(s)\right) d s\right\| \leq$ $\frac{r_{\max }+1}{2}\left\|u^{*}\right\|+\frac{c R\left\|u^{*}\right\|\left(e^{\kappa t}-1\right)}{\kappa}$. Therefore, $T_{n} \geq$ $\min \left\{\frac{1}{\kappa} \ln \left(1+\frac{\kappa\left(1-r_{\min }\right)}{2 c R}\right), \frac{1}{\kappa} \ln \left(1+\frac{\kappa\left(r_{\max }-1\right)}{2 c R}\right), T_{0}\right\}>0$.

d) For all $t \in\left(0, T^{*}\right)$ we have $\left\|u_{n}(t+\Delta t)-u_{n}(t)\right\| \leq$ $\|\Phi(t)\|_{\mathcal{L}} \cdot\left\|\Phi(\Delta t) u^{*}-u^{*}\right\|+\int_{0}^{\Delta t}\left\|\Phi(\sigma) \tilde{\xi}\left(p_{n-1}(t+\sigma), u_{n}(t+\sigma)\right)\right\| d \sigma$ $\leq\left(\frac{c R\|B K\|_{\mathcal{L}}^{\mathbf{H}}\left\|u^{*}\right\|\left(e^{\kappa \Delta t}-1\right)}{\kappa}+\left\|\Phi(\Delta t) u^{*}-u^{*}\right\|\right) \sup _{t \in\left[0, T^{*}\right]}\|\Phi(t)\|_{\mathcal{L}}$. Hence, we derive that the sequence $\left\{u_{n}\right\}$ is equicontinuous. Since the homogeneous norm $\|\cdot\|_{\mathbf{d}}$ is Lipschitz continuous on $\mathbf{H} \backslash\{\boldsymbol{0}\}$ then the sequence of scalar functions $\left\{q_{n}\right\}$ (where $q_{n}(t)=\left\|u_{n}(t)\right\|_{\mathbf{d}}$ with $t \in\left[0, T^{*}\right]$ ) is also equicontinuous. Arzela-Ascoli theorem implies that there exists a subsequence $\left\{q_{n_{k}}\right\}$ that converges uniformly on $\left[0, T^{*}\right]$ to $g \in C\left(\left[0, T^{*}\right], \mathbb{R}\right)$. Let $u_{g}$ be the corresponding solution to (20). Then for $\Delta u_{n_{k}}=u_{g}-u_{n_{k}}$ we have $\left\|\Delta u_{n_{k}}(t)\right\| \leq \int_{0}^{t}\left\|T(t-s)\left(\tilde{\xi}\left(g(s), u_{g}(s)\right)-\tilde{\xi}\left(g(s), u_{n_{k}}(s)\right)\right)\right\| d s$ $+\int_{0}^{t}\left\|T(t-s)\left(\tilde{\xi}\left(g(s), u_{n_{k}}(s)\right)-\tilde{\xi}\left(q_{n_{k}}(s), u_{n_{k}}(s)\right)\right)\right\| d s \leq$ $C(t) \sup _{s \in[0, t]}\left\|\Delta u_{n_{k}}(s)\right\|+\varepsilon_{k}(t) \sup _{s \in[0, t]}\left\|u_{n_{k}}(s)\right\|$, where $C(t)=\int_{0}^{t}\left\|T(t-s) B K g^{-\mu}(s) \mathbf{d}_{1}(-\ln g(s))\right\|_{\mathcal{L}}^{\mathbf{H}} d s$ and $\varepsilon_{k}(t)=$ $\int_{0}^{t}\left\|T(t-s) B K\left(g^{-\mu}(s) \mathbf{d}_{1}(-\ln g(s))-q_{n_{k}}^{-\mu}(s) \mathbf{d}_{1}\left(-\ln q_{n_{k}}(s)\right)\right)\right\|_{\mathcal{L}}^{\mathbf{H}} d s$.

Since $\sup _{t \in\left[0, T^{*}\right]} \varepsilon_{k}(t) \rightarrow 0$ as $n_{k} \rightarrow 0$ then for sufficiently small $t>0: C(t)<1$ we have $\lim _{k \rightarrow \infty} \sup _{s \in[0, t]}\left\|\Delta u_{k}(s)\right\|=0$ and $\left\|u_{g}(s)\right\|_{\mathbf{d}}=g(s)$ for $s \stackrel{k \rightarrow \infty}{\in}[0, t]$. Hence, we conclude that the system (1), (12) has a classical solution defined on an interval $\left[0, T\left(u^{*}\right)\right)$, where $T\left(u^{*}\right)>0$ is such that $0<\|u(t)\|<+\infty$ for all $t \in\left[0, T\left(u^{*}\right)\right)$.

III. Uniqueness of solution. Let us suppose the contrary, i.e. there exists another solution $v \in C([0, T), \mathbf{H})$ such that $v(0)=u(0)=u^{*} \in \mathcal{D}(A) \backslash\{\mathbf{0}\}$ and $u(t) \neq v(t)$ for $t \in\left(0, t^{\prime}\right]$. Since $\left\|u^{*}\right\|>0$ and $u, v$ are continuous functions of time then for sufficiently small $t^{\prime}$ we have $\|v(t)\|_{\mathbf{d}} \in\left[q_{\min }, q_{\max }\right]$ and $\|u(t)\|_{\mathbf{d}} \in\left[q_{\min }, q_{\max }\right]$ for $t \in\left[0, t^{\prime}\right]$. The evolution (20) with $g=\|v\|_{\mathbf{d}}>0$ has a unique classical solution, which coincides with $v$. This means that $v(t) \in \mathcal{D}(A)$ for all $t \in\left[0, t^{\prime}\right]$. Due to Lemma 1 the homogeneous norm is Lipschitz continuous on $\mathbf{H} \backslash\{\boldsymbol{0}\}$. Hence we derive $\|u(t)-v(t)\|=\| \int_{0}^{t} T(t-s)\left(\tilde{\xi}\left(\|u(s)\|_{\mathbf{d}}, u(s)\right)-\right.$ $\tilde{\xi}\left(\|v(s)\|_{\mathbf{d}}, v(s)\right) d s\left\|\leq \tilde{C}(t) \sup _{s \in[0, t]}\right\| u(s)-v(s) \|$, where $\tilde{C}$ is a continuous increasing function, $\tilde{C}(0)=0$, i.e. $0 \leq \tilde{C}(t)<$ 1 for $0 \leq t<t^{\prime \prime}<t^{\prime}$. Hence, we derive contradiction.

IV. Finite-time Convergence. Using formula (6) let us calculate the time derivative of homogeneous norm along the trajectory $u(t) \in \mathcal{D}(A) \quad \subset$ $\mathcal{D}\left(G_{\mathbf{d}}\right)$ of the closed-loop system (1), (12): $\frac{d}{d t}\|u(t)\|_{\mathbf{d}}=\left(D_{u}\|u(t)\|_{\mathbf{d}}\right)(\dot{u}(t))=\frac{e^{s(t)}\langle\mathbf{d}(-s(t)) \dot{u}(t), \mathbf{d}(-s(t)) u(t)\rangle}{\left\langle G_{\mathbf{d}} \mathbf{d}(-s(t)) u(t), \mathbf{d}(-s(t)) u(t)\right\rangle}$, where $s(t)=\ln \|u(t)\|_{\mathbf{d}}$, so $\|\mathbf{d}(-s(t)) u(t)\|=1$. If $u(t) \in \mathcal{D}(A)$ then due to homogeneity we have $\mathbf{d}(-s(t)) \dot{u}(t)=\mathbf{d}(-s(t))(A u(t)+B \xi(u(t))=$ $e^{-\mu s(t)}(A \mathbf{d}(-s(t)) u(t)+B K \mathbf{d}(-s(t)) u(t))$

where the condition D) has been utilized on the last step. Since $\mathbf{d}(-s(t)) u(t) \in \mathcal{D}(A)$ then due to condition $\mathrm{C})$ we obtain $\frac{d}{d t}\|u(t)\|_{\mathbf{d}} \leq-\alpha e^{(1-\mu) s(t)}=-\alpha\|u(t)\|_{\mathbf{d}}^{1-\mu}$. Therefore, $\|u(t)\|_{\mathbf{d}} \rightarrow 0$ as $t \rightarrow T \leq \frac{\|u(0)\|_{\mathbf{d}}^{\mu}}{\alpha \mu}$ for any initial condition $u(0) \in \mathcal{D}(A) \backslash\{\mathbf{0}\}$.

\section{Proof of Corollary 1}

The existence and uniqueness of solutions is proven in Theorem (1) using Conditions A)-C) only. Since due to homogeneity $\mathbf{d}(-s(t))(A u(t)+B \xi(u(t)) \in \mathbf{d}(-s(t)) F(u(t)) \subset$ $e^{-\mu s(t)} F\left(\mathbf{d}(-s(t) u(t))\right.$ and $\|\mathbf{d}(-s(t)) u(t)\|=1$ then $\left.\mathrm{D}^{*}\right)$ implies $\frac{d}{d t}\|u(t)\|_{\mathbf{d}} \leq-\alpha\|u(t)\|_{\mathbf{d}}^{1-\mu}$ (see Proof of Theorem 1).

\section{E. Proof of Corollary 2}

Since the control $\xi$ is Lipschitz continuous on $\mathbf{H} \backslash\{\boldsymbol{0}\}$ then using [35, Theorems 1.2 and 1.4, Chapter 6] we derive that for any $u_{0} \in \mathbf{H} \backslash\{\boldsymbol{0}\}$ the closed-loop system has a unique mild solution $u$ given by (14) and defined at least locally on $\left[0, T\left(u_{0}\right)\right)$ such that $u(t) \in \mathbf{H} \backslash\{\mathbf{0}\}$ if $t \in\left[0, T\left(u_{0}\right)\right)$ and $\|u(t)\| \rightarrow 0$ (or $\|u(t)\|=+\infty)$, where $T\left(u_{0}\right)$ is a finite instant of time or $T\left(u_{0}\right)=+\infty$. Since $\mathcal{D}(A)$ is dense in $\mathbf{H}$ then for any $u_{0} \in \mathbf{H} \backslash\{\mathbf{0}\}$ and any $\varepsilon>0$ there exists $u_{0}^{\mathcal{D}(A)} \in \mathcal{D}(A) \backslash\{\boldsymbol{0}\}$ such that $\left\|u_{0}-u_{0}^{\mathcal{D}(A)}\right\| \leq \varepsilon$. Let $u^{\mathcal{D}(A)}$ be a classical solution with $u^{\mathcal{D}(A)}(0)=u_{0}^{\mathcal{D}(A)} \in \mathcal{D}(A) \backslash\{\mathbf{0}\}$. Since mild solutions continuously depend of $u_{0}$ (see, [35, Theorems 1.2 and 1.4, Chapter 6]), then then $\sup _{s \in[0, t]} \| u(s)-$ $u^{\mathcal{D}(A)}(s) \| \rightarrow 0$ as $\varepsilon \rightarrow 0$ for any $t<T\left(u_{0}\right)$. Moreover, $\sup _{s \in[0, t]}\left|\|u(s)\|_{\mathbf{d}}-\left\|u^{\mathcal{D}(A)}(s)\right\|_{\mathbf{d}}\right| \rightarrow 0$ as $\varepsilon \rightarrow 0$, since $\|\cdot\|_{\mathbf{d}}$ is Lipschitz continuous on $\mathbf{H} \backslash\{\mathbf{0}\}$ and continuous at 0. We complete the proof taking into account that all classical solutions satisfy Theorem 1 (or Corollary 1) but the inequality (13) is equivalent to (15) if $u$ is a classical solutions.

\section{F. Proof of Corollary 3}

Let us denote $\mathcal{K}(\alpha, \beta)=\left\{u \in \mathcal{D}(A): \alpha<\|u\|_{\mathbf{d}} \leq \beta\right\}$. I. Existence and Uniqueness of Solution. Since $B K_{i} \in \mathcal{L}(\mathbf{H})$ for any $i \in \mathbb{Z}$, where $K_{i}=r_{i}^{-\mu} K \mathbf{d}_{1}\left(-\ln \left(r_{i}\right)\right)$, then for any initial condition $u(0)=u^{*} \in \mathcal{D}(A)$ the evolution equation

$$
\dot{u}(t)=\left(A+B K_{i}\right) u(t)
$$

has a unique classical solution $u_{i}\left(t, u^{*}\right) \in \mathcal{D}(A)$ defined for all $t \geq 0$. We have $r_{i+1}<r_{i}, i \in \mathbb{Z}$ by construction. Since $\left\|u_{i}\left(\cdot, u_{a}\right)\right\|_{\mathbf{d}}: \mathbb{R} \rightarrow \mathbb{R}_{+}$is continuous (see, property 4 on page 2) then for $u\left(t_{i}\right)=u_{0} \in \mathcal{K}\left(r_{i+1}, r_{i}\right)$ and $\sigma\left(t_{i}\right) \equiv i$ the closed-loop system (1), (2), (16), (17) has classical solution $u$ defined at least on $\left[t_{i}, t_{i+1}\right)$ and $u(t)=$ $u_{i}\left(t-t_{i}, u_{0}\right)$ for $t \in\left[t_{i}, t_{i+1}\right)$, where $t_{i+1}=+\infty$ and/or $\lim _{t \rightarrow t_{i+1}}\|u(t)\|_{\mathbf{d}}=r_{i-1}$ or $\lim _{t \rightarrow t_{i+1}}\|u(t)\|_{\mathbf{d}}=r_{i+1}$. Let us show the only latter case is possible for some $t_{i+1}<+\infty$. Since $\frac{d}{d t}\left\|\mathbf{d}\left(-\ln \left(r_{i}\right)\right) u(t)\right\|^{2}=2\left\langle\mathbf{d}\left(-\ln \left(r_{i}\right)\right) \dot{u}(t), \mathbf{d}\left(-\ln \left(r_{i}\right)\right) u(t)\right\rangle=$ $2 e^{-\mu \ln \left(r_{i}\right)}\left\langle(A+B K) \mathbf{d}\left(-\ln \left(r_{i}\right)\right) u(t), \mathbf{d}\left(-\ln \left(r_{i}\right)\right) u(t)\right\rangle \leq$ $-\frac{2 \alpha\left\langle G_{\mathbf{d}} \mathbf{d}\left(-\ln \left(r_{i}\right)\right) u(t), \mathbf{d}\left(-\ln \left(r_{i}\right)\right) u(t)\right\rangle}{r_{i}^{\mu}} \leq-\frac{2 \alpha \beta\left\|\mathbf{d}\left(-\ln \left(r_{i}\right)\right) u(t)\right\|^{2}}{r_{i}^{\mu}}$

then the continuous function $\left\|\mathbf{d}\left(-\ln \left(r_{i}\right)\right) u(\cdot)\right\|$ decays exponentially. Taking into account $\left\|\mathbf{d}\left(-\ln \left(r_{i}\right)\right) u\right\|^{2}=1$ 
if $\|u\|_{\mathbf{d}}=r_{i}$ and $\left\|\mathbf{d}\left(-\ln \left(r_{i}\right)\right) u\right\|^{2}<1$ if $\|u\|_{\mathbf{d}}<$ $r_{i}$, we derive $\|u(t)\|_{\mathbf{d}}<r_{i}$ for $t>t_{i}$ and $\lim _{t \rightarrow t_{i+1}}\|u(t)\|_{\mathbf{d}}=r_{i+1}$. Moreover, $t_{i+1} \leq t_{i}+$ $r_{i}^{\mu} \beta^{-1} \alpha^{-1} \ln \left(\frac{\left\|\mathbf{d}\left(-\ln \left(r_{i}\right)\right) u\left(t_{i}\right)\right\|}{\varepsilon_{i}}\right)$, where $\varepsilon_{i}>0$ is such that $\left\|\mathbf{d}\left(-\ln \left(r_{i}\right)\right) u\right\| \leq \varepsilon_{i} \Rightarrow\|u\|_{\mathbf{d}} \leq r_{i+1}$.

Therefore, $u\left(t_{i+1}\right)=u_{i}\left(t_{i+1}-t_{i}, u_{0}\right) \in \overline{\mathcal{D}}(A)$. Repeating the same considerations for $\left[t_{i+1}, t_{i+2}\right]$, etc, and taking into account uniqueness and continuity of solutions of the equation (*) we derive that for any initial condition $u(0) \in \mathcal{D}(A) \backslash\{0\}$, $\sigma(0)=i_{0}$ such that $r_{i_{0}+1}<\|u(0)\|_{\mathbf{d}}<r_{i_{0}-1}$ the closedloop system (1), (2), (16), (17) has a unique strong solution defined on $\left[0, T\left(u_{0}\right)\right)$, which may not have time derivative only at isolated instances of time $t_{i}:\left\|u\left(t_{i}\right)\right\|_{\mathbf{d}}=r_{i}$, where $T\left(u_{0}\right)=\sum_{j=i_{0}}^{+\infty} t_{i+1}-t_{i}$ is the unique accumulation point of the set $\left\{t_{i}\right\}$. In addition, $\sigma(t)=$ const for $t \in\left(t_{i}, t_{i+1}\right]$. Since $\sum_{i=0}^{+\infty} q_{i}=+\infty$ then $r_{i}=\frac{1}{e^{\sum_{j=0}^{i} q_{j}}} \rightarrow 0$ as $i \rightarrow+\infty$ and $\|u(t)\| \rightarrow 0$ as $t \rightarrow T\left(u_{0}\right)$.

II. Settling Time Estimate. Taking into account $\beta\|\mathbf{d}(s) u\|^{2} \leq$ $\left\langle G_{\mathbf{d}} \mathbf{d}(s) u, \mathbf{d}(s) u\right\rangle=\frac{1}{2} \frac{d}{d s}\|\mathbf{d}(s) u\|^{2}$ we derive $\|\mathbf{d}(s) u\| \geq e^{\beta s}\|u\|$ and $\left\|\mathbf{d}\left(-\ln \left(r_{i}\right)\right) u\right\|=\left\|\mathbf{d}\left(\ln \left(\|u\|_{\mathbf{d}}\right)-\ln \left(r_{i}\right)\right) \mathbf{d}\left(-\ln \left(\|u\|_{\mathbf{d}}\right)\right) u\right\|$ $\geq e^{\beta \ln \left(\|u\|_{\mathbf{d}} / r_{i}\right)}=\|u\|_{\mathbf{d}}^{\beta} / r_{i}^{\beta}$. Selecting $\varepsilon_{i}=e^{-\beta q_{i}}$ we obtain $t_{i+1} \leq t_{i}+\alpha^{-1} r_{i}^{\mu} q_{i}$ and the settling time $T\left(u_{0}\right)$ is finite since $\lim _{i \rightarrow+\infty} r_{i+1}^{\mu} q_{i+1} /\left(r_{i}^{\mu} q_{i}\right)=\lim _{i \rightarrow+\infty} q_{i+1} e^{-\mu q_{i}} / q_{i}<1$.

III. Boundedness of Control. Since for $u(t) \in \mathcal{K}\left(r_{i+1}, r_{i}\right)$ and $\sigma(t)=i$ the control law can be rewritten as follows $\xi_{s w}(t)=r_{i}^{-\mu} K \mathbf{d}_{1}\left(-\ln r_{i}\right) u(t)$. Taking into account (5), $\|\mathbf{d}(s)\|_{\mathcal{L}}^{\mathbf{H}} \leq e^{\beta s}$ and $\left\|\mathbf{d}_{1}(s)\right\|_{\mathcal{L}}^{\mathbf{H}} \leq e^{\gamma s}$ if $s \leq 0$, but $\|\mathbf{d}(s)\|_{\mathcal{L}}^{\mathbf{H}} \leq$ $M e^{(\mu+\gamma) s}$ with $M \geq 1$ and $\left\|\mathbf{d}_{1}(s)\right\|_{\mathcal{L}}^{\mathbf{H}} \leq e^{\left\|G_{\mathbf{d}_{1}}\right\|_{\mathcal{L}}^{\mathbf{H}} s}$ if $s \geq 0$ we


$\beta-\mu-\left\|G_{\mathbf{d}}\right\|_{\mathcal{L}}^{\mathbf{H}} \geq 0$ (see Theorem 1).

\section{REFERENCES}

[1] V. Zubov, "On systems of ordinary differential equations with generalized homogenous right-hand sides," Izvestia vuzov. Mathematica., vol. 1, pp. 80-88, 1958 (in Russian).

[2] V. V. Khomenuk, "On systems of ordinary differential equations with generalized homogenous right-hand sides," Izvestia vuzov. Mathematica., vol. 3(22), pp. 157-164, 1961 (in Russian).

[3] W. Hahn, Stability of Motion. New York: Springer-Verlag Berlin Heidelberg, 1967.

[4] H. Hermes, "Nilpotent approximations of control systems and distributions," SIAM Journal of Control and Optimization, vol. 24, no. 4, p. 731, 1986.

[5] L. Rosier, "Homogeneous Lyapunov function for homogeneous continuous vector field," Systems \& Control Letters, vol. 19, pp. 467-473, 1992.

[6] - "Etude de quelques problemes de stabilization," PhD Thesis, Ecole Normale Superieure de Cachan (France), 1993.

[7] M. Kawski, "Geometric homogeneity and stabilization," in Proc. IFAC Nonlinear Control Symposium, A. Krener and D. Mayne, Eds., Lake Tahoe, CA, 1995, pp. 164-169.

[8] V. I. Zubov, Methods of A.M. Lyapunov and Their Applications. Noordhoff, Leiden, 1964.

[9] A. Bacciotti and L. Rosier, Lyapunov Functions and Stability in Control Theory. Springer, 2001.

[10] J.-M. Coron and L. Praly, "Adding an integrator for the stabilization problem," Systems \& Control Letters, vol. 17, no. 2, pp. 89-104, 1991.

[11] S. P. Bhat and D. S. Bernstein, "Geometric homogeneity with applications to finite-time stability," Mathematics of Control, Signals and Systems, vol. 17, pp. 101-127, 2005.

[12] Y. Orlov, "Finite time stability and robust control synthesis of uncertain switched systems," SIAM Journal of Control and Optimization, vol. 43, no. 4, pp. 1253-1271, 2005.
[13] V. Andrieu, L. Praly, and A. Astolfi, "Homogeneous Approximation, Recursive Observer Design, and Output Feedback," SIAM Journal of Control and Optimization, vol. 47, no. 4, pp. 1814-1850, 2008.

[14] A. Levant, "Homogeneity approach to high-order sliding mode design," Automatica, vol. 41, no. 5, pp. 823-830, 2005.

[15] A. Polyakov, D. Efimov, and W. Perruquetti, "Finite-time and fixed-time stabilization: Implicit lyapunov function approach," Automatica, vol. 51, no. 1, pp. 332-340, 2015.

[16] W. Perruquetti, T. Floquet, and E. Moulay, "Finite-time observers: application to secure communication," IEEE Transactions on Automatic Control, vol. 53, no. 1, pp. 356-360, 2008.

[17] V. Andrieu, L. Praly, and A. Astolfi, "High-gain observers with updated high-gain and homogeneous correction terms," Automatica, vol. 45, no. 2, pp. 422-428, 2009.

[18] F. Lopez-Ramirez, A. Polyakov, D. Efimov, and W. Perruquetti, "FiniteTime and Fixed-Time Observers Design via Implicit Lyapunov Function," in European Control Conference (ECC), 2016.

[19] E. Ryan, "Universal stabilization of a class of nonlinear systems with homogeneous vector fields," Systems \& Control Letters, vol. 26, pp. 177-184, 1995.

[20] Y. Hong, " $\mathrm{H}_{\infty}$ control, stabilization, and input-output stability of nonlinear systems with homogeneous properties," Automatica, vol. 37, no. 7, pp. 819-829, 2001.

[21] E. Bernuau, A. Polyakov, D. Efimov, and W. Perruquetti, "Verification of ISS, iISS and IOSS properties applying weighted homogeneity," System \& Control Letters, vol. 62, no. 12, pp. 1159-1167, 2013.

[22] A. Polyakov, D. Efimov, E. Fridman, and W. Perruquetti, "On homogeneous distributed parameters equations," IEEE Transactions on Automatic Control, vol. 61, no. 11, pp. 3657-3662, 2016.

[23] V. Korobov, Method of Controlability Functions (in Russian). NIC, Moscow, 2007.

[24] V. Perrollaz and L. Rosier, "Finite-time stabilization of $2 \times 2$ hyperbolic systems on tree-shaped networks," SIAM Journal of Control and Optimization, vol. 52, no. 1, pp. 143-163, 2014.

[25] J.-M. Coron, L. Hu, and G. Olive, "Stabilization and controllability of first-order integro-differential hyperbolic equations," Journal of Functional Analysis, vol. 271, no. 12, pp. 3554-3587, 2016.

[26] J.-M. Coron and H.-M. Nguyen, "Null controllability and finite time stabilization for the heat equations with variable coefficients in space in one dimension via backstepping approach," Archive for Rational Mechanics and Analysis, 2017 (doi:10.1007/s00205-017-1119-y).

[27] L. Hu and F. Di Meglio, "Finite-time backstepping boundary stabilization of $3 \times 3$ hyperbolic systems," in European Control Conference (ECC), 2015, pp. 67-73.

[28] M. Tucsnak and G. Weiss, Observation and Control for Operator Semigroups. Birkhauser, 2009.

[29] Y. Orlov, "Application of Lyapunov method in distributed systems," Automation and Remote Control, vol. 44, pp. 426-430, 1983.

[30] A. Pisano, Y. Orlov, and E. Usai, "Tracking control of the uncertain heat and wave equation via power-fractional and sliding-mode techniques," SIAM Journal on Control and Optimization, vol. 49, no. 2, pp. 363-382, 2011.

[31] V. Korobov, "A general approach to synthesis problem," Doklady Academii Nauk SSSR, vol. 248, pp. 1051-1063, 1979.

[32] J. Adamy and A. Flemming, "Soft variable-structure controls: a survey," Automatica, vol. 40, pp. 1821-1844, 2004.

[33] A. Polyakov, D. Efimov, and W. Perruquetti, "Robust stabilization of mimo systems in finite/fixed time," International Journal of Robust and Nonlinear Control, vol. 26, no. 1, pp. 69-90, 2016.

[34] A. Polyakov, J.-M. Coron, and L. Rosier, "On finite-time stabilization of evolution equations: A homogeneous approach," in Conference on Decision and Control, 2016, pp. 3143-3148.

[35] A. Pazy, Semigroups of Linear Operators and Applications to Partial Differential Equations. Springer, 1983.

[36] D. Efimov and W. Perruquetti, "Oscillations conditions in homogenous systems," in Proc. IFAC NOLCOS Symp., 2010, pp. 1379-1384.

[37] D. Liberzon, Switchings in Systems and Control. Birkhauser, Boston, 2003.

[38] A. Polyakov, J.-M. Coron, and L. Rosier, "Boundary finite-time feedback design for heat equation," in IFAC World Congress, 2017.

[39] M. Krstic and A. Smyshlyaev, Boundary Control of PDEs: A Course on Backstepping Designs. SIAM, 2008.

[40] H. Halkin, "Implicit functions and optimization problems without continuous differentiability of the data." SIAM Journal of Control and Optimization, vol. 12, no. 2, 1974. 